\title{
Maternal exposure to polystyrene nanoplastics causes brain abnormalities in progeny
}

\section{Bohyeon Jeong}

Korea Research Institute of Bioscience and Biotechnology (KRIBB)

\section{Young-Kyoung Ryu}

Korea Research Institute of Bioscience and Biotechnology (KRIBB)

Jeong Yeob Baek

Korea Research Institute of Bioscience and Biotechnology (KRIBB)

Jahong Koo

Korea Research Institute of Bioscience and Biotechnology (KRIBB)

\section{Subin Park}

Korea Research Institute of Bioscience and Biotechnology (KRIBB)

\section{Seungjae Zhang}

Konkuk University

\section{ChiHye Chung}

Konkuk University

\section{Rumeysa Dogan}

Pohang University of Science and Technology (POSTECH)

\section{Hyung-Seok Choi}

Pohang University of Science and Technology (POSTECH)

\section{Dahun Um}

Pohang University of Science and Technology (POSTECH)

\section{Tae-Kyung Kim}

Pohang University of Science and Technology (POSTECH)

\section{Wang Sik Lee}

Korea Research Institute of Bioscience and Biotechnology (KRIBB)

\section{Kyoung-Shim Kim}

Korea Research Institute of Bioscience and Biotechnology (KRIBB)

Jinyoung Jeong

Korea Research Institute of Bioscience and Biotechnology (KRIBB)

\section{Won-Ho Shin}

Korea Institute of Toxicology

Jae-Ran Lee

Korea Research Institute of Bioscience and Biotechnology (KRIBB) 
Korea Research Institute of Bioscience and Biotechnology (KRIBB)

Da Yong Lee ( $\nabla$ daylee@kribb.re.kr)

Korea Research Institute of Bioscience and Biotechnology (KRIBB)

\section{Article}

Keywords: polystyrene nanoplastics, neurodevelopmental defects, maternal exposure

Posted Date: January 20th, 2021

DOl: https://doi.org/10.21203/rs.3.rs-131249/v1

License: (c) (i) This work is licensed under a Creative Commons Attribution 4.0 International License. Read Full License

Version of Record: A version of this preprint was published at Journal of Hazardous Materials on November 1st, 2021. See the published version at https://doi.org/10.1016/j.jhazmat.2021.127815. 
Authors: Bohyeon Jeong ${ }^{1,2}$, Young-Kyoung Ryu ${ }^{3}$, Jeong Yeob Baek ${ }^{1}$, Jahong Koo ${ }^{1,2}$, Subin Park ${ }^{1}$, Seungjae Zhang ${ }^{4}$, ChiHye Chung ${ }^{4}$, Rumeysa Dogan ${ }^{5}$, Hyung-Seok Choi ${ }^{5}$, Dahun Um ${ }^{5}$, Tae-Kyung $\mathrm{Kim}^{5}$, Wang Sik Lee ${ }^{6}$, Kyoung-Shim Kim³ ${ }^{3}$ Jinyoung Jeong ${ }^{6,7}$, Won-Ho Shin ${ }^{8}$, Jae-Ran Lee ${ }^{1}$, NamSoon $\mathrm{Kim}^{1}$, and Da Yong Lee $\mathrm{Le}^{1,2 *}$

\section{8 - Institutions:}

1. Rare Disease Research Center, Korea Research Institute of Bioscience and Biotechnology (KRIBB), 125 Gwahak-ro, Yuseong-gu, Daejeon 34141, South Korea.

2. Department of Functional Genomics, KRIBB School of Bioscience, University of Science and Technology, Daejeon, South Korea.

3. Laboratory animal Resource Center, Korea Research Institute of Bioscience and Biotechnology (KRIBB), 125 Gwahak-ro, Yuseong-gu, Daejeon 34141, South Korea.

4. Department of Biological Sciences (Neurophysiology Laboratory, C-Lab), Konkuk University, 120 Neungdong-ro, Gwangjin-gu, Seoul 05029, South Korea.

5. Department of Life Sciences, Pohang University of Science and Technology (POSTECH), Pohang, South Korea.

6. Environmental Disease Research Center, Korea Research Institute of Bioscience and Biotechnology (KRIBB), 125 Gwahak-ro, Yuseong-gu, Daejeon 34141, South Korea.

7. Department of Nanobiotechnology, KRIBB School of Biotechnology, University of Science and Technology, Daejeon, South Korea.

8. Department of Predictive Toxicology, Korea Institute of Toxicology, Daejeon 34114, South Korea. 
26 *Address correspondence to: Da Yong Lee, PhD, Rare Disease Research Center, Korea Research

27 Institute of Bioscience and Biotechnology (KRIBB), 125 Gwahak-ro, Yuseong-gu, Daejeon 34141,

28 South Korea. 82-42-860-4475 (Phone); 82-42-879-8495 (FAX); daylee@kribb.re.kr (E-mail).

29 
31 As global plastic production continues to grow, microplastics released from a massive quantity of 32 plastic wastes have become a critical environmental concern. These microplastic particles are found in 33 a wide range of living organisms in a diverse array of ecosystems. In this study, we investigated the 34 biological effects of polystyrene nanoplastics (PSNPs) on development of the central nervous system 35 using cultured neural stem cells (NSCs) and mice exposed to PSNPs during developmental stages. 36 Our study demonstrates that maternal administration of PSNPs during gestation and lactating periods 37 altered the functioning of NSCs, neural cell compositions, and brain histology in progeny. Similarly, our 38 in vitro study also shows PSNP-induced molecular and functional defects in NSCs. Finally, we show that the abnormal brain development caused by exposure to high concentrations of PSNPs results in 40 neurophysiological and cognitive deficits in a gender-specific manner. Our data demonstrate the 41 possibility that exposure to high amounts of PSNPs may increase the risk of neurodevelopmental 42 defects. 
Continuous increases in global plastic consumption have resulted in the generation of a massive amount of plastic waste that finally ends up in their release and potential accumulation in the environment as tiny invisible plastic particles. These tiny plastic particles are defined as either microplastics $(<5 \mathrm{~mm})$ or nanoplastics $(<1 \mu \mathrm{m})$ on the basis of their diameters ${ }^{1}$. Besides the secondary microplastics generated from degradation of a large mass of plastic waste through the process of weathering, primary micro- and nanoplastics are often purposely manufactured for a wide range of applications $^{2-5}$. Increasing evidence shows that when they are released into the environment, these tiny plastic particles infiltrate various living organisms, particularly in the oceans, and induces toxic effects including cytotoxicity, oxidative stress, metabolic changes, and locomotion defects ${ }^{6-8}$. Moreover, microplastic particles are not only detected in aqueous environments, but also in a broad range of terrestrial $(0.54-67,500 \mathrm{mg} / \mathrm{kg} ; 1.3-42,960 \text { items } / \mathrm{kg} \text { of soil })^{9}$ and atmospheric $(0-11,130$ particles $/ \mathrm{m}^{2} /$ day) ecosystems ${ }^{10}$. Humans are at the top of the food chain; therefore, it is possible that the amount of microplastic absorbed by humans could be higher than that absorbed by other organisms. In this regard, a recent study reported that humans can absorb considerable amounts (approximately 203-312 particles/day, 74,060-121,664 particles/year) of microplastics from the environment on a daily basis through food ingestion and other routes such as inhalation and dermal exposure (0.01-66.81 $\mathrm{g} /$ day $)^{11}$. In rodents, orally administered microplastics $(0.1-1 \mathrm{mg} /$ day $)$ can accumulate in various organs and finally cause metabolic disorders, oxidative stress, neurotoxicity, and reduced reproduction ${ }^{12,13}$. In terms of the relationship between the size of plastic particles and toxicity, smaller particles have higher toxicity ${ }^{14,15}$, although the majority of nanoplastics in the environment remain difficult to detect because of technical barriers ${ }^{16,17}$.

Recently, environmental risks that may cause brain developmental disorders have become a critical concern, as the number of children with neurodevelopmental disorders continues to increase ${ }^{18}$. Compared with adults, infants and young children are far more vulnerable, as the brain and other organs are still developing. Moreover, in mammals, maternally-ingested toxic materials could possibly infiltrate the brain of progeny through breast milk, as the blood brain barrier (BBB) is not fully matured in neonatal developmental stages ${ }^{19,20}$. Regarding the effects of micro- and nano-sized plastics on CNS development, exposure to plastic particles causes locomotion defects in zebrafish and mice 21,22 . 
73 Moreover, polypropylene microplastics (5-10 $\mu \mathrm{m}$ diameters) and silica nanoparticles (35-70 $\mathrm{nm}$ 74 diameter) are directly delivered from pregnant females to fetal organs such as blood capillaries, the 75 liver, and the brain through trophoblast layers and the placenta during embryogenesis ${ }^{23}$.

76 In this study, we examined whether exposure to polystyrene nanoplastic (PSNP) particles via maternal 77 administration during embryonic and early postnatal stages affects neurodevelopment in mice. 78 Furthermore, we also examined the effects of PSNPs on primary mouse neural stem cells (NSCs). We 79 found that maternally-administered PSNPs delivered to progeny led to histological changes in the brain 80 of the progeny at postnatal stages. Using primary NSCs, we demonstrated that PSNPs induced 81 abnormal changes in the proliferation, multi-lineage differentiation potential, and gene expression 82 pattern of NSCs. Finally, we showed that PSNP-induced abnormal brain development resulted in neurophysiological defects and cognitive deficits, especially in female mice. 
In this study, we sought to investigate the effect of PSNPs on brain development using yellow-green fluorescence (YG)-conjugated carboxylated PSNPs (50 nm diameter) in mice. To determine the effects of PSNPs on brain development, PSNPs were orally administered to pregnant and lactating female mice and the brain histology, neurophysiology, and behavior of the progeny were analyzed in the postnatal stages (Fig. 1a). As the majority of forebrain regions and maternal mammary glands start development around 8-10 days after fertilization ${ }^{24}$, PSNPs were administered from embryonic day 8 (E8) until 2 weeks after birth (Supplementary Fig. 1). The effects of PSNPs were evaluated using a broad range of doses (0.5-1,000 $\mu \mathrm{g} /$ day) according to previous rodent studies (Supplementary Table. 1). Scanning electron microscopy (SEM) showed that the PSNPs had an appropriate diameter of approximately $50 \mathrm{~nm}$ and spherical shapes (Supplementary Fig. 2a). For oral administration and dosedependency analysis of the effects of PSNPs on the brain, agarose jelly cubes containing various amounts of YG-PSNPs $\left(0-1,000 \mu \mathrm{g} / \mathrm{cm}^{3}\right.$ cube) were prepared. Under both white light and fluorescence microscopy, the YG fluorescence intensity of the jelly cubes increased in association with the amount of YG-PSNPs (Supplementary Fig. 2b). After maternal exposure, YG signals were detected in various brain regions (sagittal view) of the postnatal progeny (P7) including the olfactory bulb, hypothalamus, with the highest intensity being detected in the hippocampus and cerebellar purkinje cell layer. However, YG signal intensity in the brainstem was far lower than in other regions. The signal intensity from the YG-PSNPs increased in a dose-dependent manner in the hippocampus and the cerebellum (Supplementary Fig. 2c).

To investigate the detailed absorption route and the distribution of PSNPs in the mothers and progeny at various developmental stages, we analyzed the YG signal in organs of both mothers and progeny at various time points after maternal administration of YG-PSNPs (500 $\mu \mathrm{g} /$ day). We found no YG signal in any organs of the embryos (E14) (Fig. 1b), or in the umbilical cord (Fig. 1c). Similarly, we observed very little YG signal in the fetal side of the placenta (labyrinth), whereas clear YG signals were detected in the maternal side of the placenta (decidua; Fig. 1c). These data suggest that maternally-administered PSNPs may not be directly absorbed by the embryo during gestation. To examine whether maternally ingested PSNPs are delivered to the progeny during neonatal and early postnatal stages, we first analyzed YG signal in the organs of lactating females and found high YG signal intensity in the 
intestines, blood, and mammary glands (Fig. 1d). We next analyzed the organs of postnatal progeny at $P 1$ and $P 7$, and clearly found $Y G$ signals in the intestines, blood, and brain regions, including the hippocampus and cortex (Fig. 1e). Collectively, these data demonstrate that maternally-administered PSNPs were delivered to progeny through breast milk after birth, rather than being directly delivered to embryos during gestation (Fig. 1a).

\section{Maternal administration of PSNPs alters the body and brain weights of offspring.}

To determine the effects of PSNPs on development, we first analyzed the body and brain weights of the progeny at various developmental stages after maternal administration of PSNPs (from E8 to 2 weeks after birth; Fig. 2a). At 1 week after birth (P7), the body weights in the groups exposed to 100 and $500 \mu \mathrm{g}$ of PSNPs daily were clearly higher than in the control group (Fig. 2b and Supplementary Table. 2). Similarly, the groups exposed to 10,100 , and $500 \mu \mathrm{g}$ of PSNPs daily also showed slightly higher brain weights than the control group (Fig. 2b). However, we did not observe an increase in either body weight or brain weight in the pups exposed to the highest dose (1,000 $\mu \mathrm{g}$ PSNPs/day). The group exposed to $1,000 \mu \mathrm{g}$ PSNPs/day showed a decrease in body weight compared with the controls (Fig. 2b). This inconsistency at the highest dose could be a result of toxicity from the excessive amounts of PSNPs, even though no lethality was observed in any of the groups until 11-12 weeks after birth (data not shown). A further analysis of body weights in the postnatal individuals showed that the most significant increases in body weight occurred after administration of 100-500 $\mu$ g PSNPs/day until 6 weeks after birth, with the highest increase at 6 weeks regardless of gender (Fig. 2c and Supplementary Table. 2). By contrast, no change was found in the weight or length of the embryos at E14 (Fig. 2d). Collectively, these data show that maternally-administered PSNPs abnormally altered body and brain weights of progeny in the postnatal stages.

\section{PSNPs induce histological changes in the developing brain.}

To investigate the effects of PSNPs on brain development, we performed histological analysis of the brains of the progeny at $1 \mathrm{w}$ after maternal administration of PSNPs, using specific markers for various 
neural cell types (Supplementary Fig. 1). The YG signal was clearly detectable in the hippocampus after PSNP exposure (Fig. 3a). As NSCs, which have self-renewal and multi-lineage differentiation potential, are the most essential cell type for brain development, we first examined whether NSCs in brain stem cell niches were altered by PSNPs. Exposure to high doses of PSNPs (500-1,000 $\mu \mathrm{g} /$ day) profoundly reduced (more than a $60 \%$ reduction) the number of Ki67+ proliferative cells in the hippocampus. In addition, progenitor cells positively labeled with nestin, a specific marker for NSCs, were also lower in the hippocampus at high doses of PSNPs (500-1,000 $\mu \mathrm{g} / \mathrm{day}$; Fig. 3a). Conversely, in the subventricular zone (SVZ) near to the lateral ventricles, we found no YG signal (Supplementary Fig. 3a) and no alteration in Ki67 or nestin immunostaining of NSCs after PSNP administration (Supplementary Fig. 3b). By genotyping of the gender with SRY primer, we confirmed that there was no gender specificity in the PSNP-induced lowering of proliferative NSCs in the hippocampus (Supplementary Fig. 4). These data clearly show that exposure to PSNPs alters the functioning of NSCs in the specific brain regions.

As the brain weight and proliferative NSCs in the hippocampus were clearly altered in the group treated with $500 \mu \mathrm{g} /$ day PSNPs, we used $500 \mu \mathrm{g} /$ day for the following analyses of neurological effects. To examine whether PSNPs alter the grey matter (which is composed of neuronal cell bodies) of the brain, we used NeuN, a marker of neuronal nuclei. We found that NeuN+ cells were lower in the hippocampal striatum radiatum and lacunosum-moleculare of PSNP-exposed mice (500 $\mu \mathrm{g} /$ day; Fig. 3b). Moreover, both NeuN immunolabeling and hematoxylin and eosin (H\&E) staining results showed that the thickness of the neuronal layer in the hippocampal CA3 region was clearly lower in the mice exposed to PSNPs, whereas no change was observed in the CA1 and dentate gyrus of the hippocampus (Fig. 3c). Next, to investigate the effects of PSNPs on neuronal axons in white matter of the brain, we analyzed the histology of the corpus callosum, the largest white matter tract in the brain. Besides the neuronal soma, the thickness of the corpus callosum was also clearly lower in both the medial and lateral hemispheric regions of PSNP-exposed mice (Fig. 3d). Additionally, the number of GFAP+ astrocytes in white matter, including the corpus callosum and internal capsule, was remarkably higher in mice exposed to PSNPs than in control mice (Fig. 3e). Collectively, these data demonstrate that exposure to PSNPs during development alters not only NSC functionality, but also the number and functions of differentiated neurons and glia in the developing brain. 
PSNPs cause functional abnormalities in hippocampal NSCs.

172 For a detailed investigation of the biological effects of PSNPs on NSC function, we chose cultured primary hippocampal NSCs as an in vitro model. As brain NSCs are proliferative multipotent stem cells, we examined the effects of PSNPs on NSC proliferation and multi-lineage differentiation ability (Fig. 4a) using carboxylated PSNPs (50 nm; Fig. 4) and large carboxylated (500 nm) PSNPs and plain PSNPs (50 and $500 \mathrm{~nm}$ ) as well (Supplementary Fig. 5 and 6). We first used SEM imaging to confirm that all PSNP particles showed a suitable range of diameters (Supplementary Fig. 5a). Then, we examined whether the PSNP particles were evenly scattered through the culture media without aggregation. Our dynamic light scattering (DLS) analysis data clearly show that all four PSNP types had appropriate diameters without any aggregation in distilled water (DW) or the culture media used for both proliferation and differentiation until day 7 after treatment (Supplementary Fig. 5b). Next, we analyzed the effects of PSNPs on NSC proliferation at various doses $(0-100 \mu \mathrm{g} / \mathrm{ml})$ using YGconjugated carboxylated PSNPs (50 nm diameter). We found lower total cell numbers at $\geq 10 \mu \mathrm{g} / \mathrm{ml}$ PSNPs (Fig. 4b) with no significant cell death (data not shown). On the basis of these results, we used $25 \mu \mathrm{g} / \mathrm{ml}$ PSNPs for the rest of our in vitro experiments because we found a clear reduction in proliferation without a cytotoxic effect at this dose.

In a detailed analysis of NSC proliferation, we found lower levels of Ki67+ proliferative cells in all four groups treated with the different types of PSNPs, with the most significant difference occurring in the group with $50 \mathrm{~nm}$ carboxylated PSNPs (Fig. 4c and Supplementary Fig. 6a). Moreover, the sizes of the neurospheres and the total numbers of cells were clearly lower in all four PSNP-exposed groups than in the controls (Fig. 4d and Supplementary Fig. 6b), although nestin was well expressed in all five groups (Fig. 4c and Supplementary Fig. 6a). To ensure that our experimental results were not limited to products from a specific company, we repeated our experiments with PSNPs $(30 \mathrm{~nm})$ from another company and obtained consistent results, as shown in Supplementary Fig. 7a-d. lower in those exposed to PSNPs in vitro (Fig. 4e and Supplementary Fig. 6c and 7e). Similar to our in vivo data, substantially more cells differentiated into GFAP+ astrocytes following in vitro exposure to 
PSNPs of all types (Fig. 4e and Supplementary Fig. $6 \mathrm{c}$ and 7e). Altogether, these data demonstrate that nanometer-sized PSNP particles lead to lower NSC proliferation and alteration of both neurogenesis and gliogenesis in the hippocampus.

\section{The transcriptome pattern in mouse hippocampal NSCs was altered by PSNPs}

To investigate the effects of PSNPs on neural development at a molecular level, we performed transcriptome analysis using mouse hippocampal NSCs exposed to $50 \mathrm{~nm}$ carboxylated PSNPs. Two independent replicates were analyzed for changes at the transcriptome level. Using $p<0.05$ and 1.5fold change (FC) filtering, we found more prominent transcriptional down-regulation than up-regulation in NSCs exposed to PSNPs (Fig. 5a). Gene ontology (GO) analysis showed that the majority of commonly down-regulated genes belong to the pathways related to cell division and proliferation. Many genes in this group serve as a hub, forming extensive protein-protein interactions with other proteins involved in cell cycle regulation (Fig. 5b, c). Of note, several kinases (e.g., Aurka/b, Bub1, and Plk1) and regulatory proteins (e.g., Cdc20, Ccna2, and Ccnb1) that are critical for cell cycle progression were identified as the major interaction hubs within the network formed by the PSNP-sensitive genes (Fig. 5c). This result collectively demonstrates that the PSNP-induced defects in the proliferation of NSCs and neurogenesis in the hippocampus are caused by selective down-regulation of the genes that coordinate to control cell division and proliferation.

Regarding the PSNP-induced increase in astrocytes observed in vivo and in vitro (Fig. $3 \mathbf{e}$ and 4e), we found lower mRNA expression of neurogranin (Nrgn) after NSCs were exposed to PSNPs (Fig. 5d). This observation is consistent with a previous report, which showed the number of GFAP+ astrocytes was higher in the hippocampus of Nrgn-deficient mice than in that of wild type mice ${ }^{25}$. In addition, we found increased mRNA expression of platelet-derived growth factor receptor $\beta$ (Pdgfrb), which has implications for astrocyte reactivity and astroglioma formation ${ }^{26,27}$, after PSNP exposure (Fig. 5d). Taken together, the PSNP-induced abnormal increase in astrocytes could be caused by altered expression of Nrgn and Pdgfrb genes in the developing brain. 

dependent manner.

227 We next sought to examine whether PSNP-induced abnormal changes in brain structures and NSC functions were associated with functional abnormalities of the brain in adulthood by analyzing synaptic plasticity, neurotransmitters, and behavior after maternal exposure to PSNPs.

First, we investigated whether exposure to PSNP alters synaptic plasticity in the hippocampus. When long-term potentiation (LTP) was induced in the Schaffer collateral pathway of the hippocampus using theta burst stimulation (TBS), we found that LTP remained intact in both male and female mice after PSNP exposure (Supplementary Fig. 8a). The magnitude of the LTP did not differ between control and PSNP-exposed groups in either sex (Supplementary Fig. 8a; males, $p>0.8$; females, $p>0.9$ ). However, when low frequency stimulation (LFS; a well-known stimulation protocol for long term depression (LTD) induction) was given, it failed to induce any significant decrease in field excitatory postsynaptic potential (fEPSP) slopes in the PSNP-exposed groups (Fig. 6a and Supplementary Fig. 8a). This LTD induction failure was common to both genders when the baseline fEPSP slopes were compared with those 30 min after LFS (Fig. 6a). The magnitude of LTD was significantly different in PSNP-exposed female mice in comparison with controls, suggesting that the impact of PSNP exposure on bidirectional synaptic plasticity may qualitatively differ according to gender (Fig. 6a).

Second, to examine whether PSNPs alter neurotransmitters in the brain, we measured the amount of glutamate and GABA in the hippocampus and the cortex following exposure to PSNPs. After PSNP administration, we found $20 \%$ higher GABA in the hippocampus of female mice in comparison with controls, but did not find alteration of GABA in male mice. However, we found no change in glutamate in either the hippocampus or the cortex after PSNP administration, irrespective of gender (Fig. $\mathbf{6 b}$ and Supplementary Fig. 8b).

Third, to examine whether PSNP-induced neurophysiological alterations eventually lead to behavioral deficits, we investigated the effect of PSNPs on cognitive function using a Y-maze test and a novel object recognition test (NOT; Fig. 6c and Supplementary Fig. 9). The results of the Y-maze test demonstrated that only female mice exposed to PSNPs, but not male mice, had a lower alternation rate $(8 \%)$ than the controls (Fig. 6c). Similarly, the NOT results also showed that only female progeny 
253 exposed to PSNPs had a reduction in both exploration time (42\%) and frequency (38\%) in comparison

254 with the control group (Fig. 6c). However, no change was found in locomotion or social interaction after 255 PSNP administration (Fig. 6c and Supplementary Fig. 9). Taken together, these data suggest the 256 possibility that exposure to high amounts of PSNPs during developmental stages may increase the risk 257 of brain dysfunction and cognitive deficit, especially in female mice. 
260 The current study demonstrates that PSNPs induce abnormalities in NSC functioning and brain development that ultimately result in neuronal dysfunction and cognitive deficit. Since the exact amount of microplastics consumed by people on a daily (or annual) basis is still unknown, we examined the effects of a wide range of doses of PSNPs according to previous studies (Supplementary table 1). The results demonstrated that exposure to large doses of PSNPs ( $\geq 500 \mu \mathrm{g} /$ day) caused significant abnormalities in brain development, whereas low doses of PSNPs did not, suggesting that high doses of PSNPs may increase the risk of neurodevelopmental defects. In vivo and in vitro experimental approaches showed that PSNPs could increase the risk of cognitive deficit, but not that of other neurological defects such as locomotive and emotional defects. This is in contrast to previous studies showing that intake of a different type of microplastic, polyethylene microplastic, leads to anxiety and locomotion deficit in mice ${ }^{22}$, suggesting that the action mechanisms and biological effects of microplastics may differ depending on the type of microplastic.

In this study, we found that maternally-administered PSNPs were delivered to progeny through breast milk at early postnatal stages, rather than directly to the embryos during pregnancy. This finding is consistent with previous studies showing that the placental barrier blocks polystyrene transmission to the fetus, regardless of the size or surface charge of the particles ${ }^{28}$. By contrast, polypropylene, silica, and titanium dioxide nanoparticles can pass through the placental barrier and directly infiltrate fetal organs ${ }^{15,29}$. This discrepancy could be due to differences in the properties of each nanoparticle type. Although there was no direct transmission of particles to the embryos, we commenced PSNP treatment at E8, rather than in the neonatal stages, to ensure strong accumulation of PSNPs in the mammary glands of the pregnant females, as the mammary gland undergoes development from approximately 89 days after fertilization ${ }^{24}$. In this regard, our experimental design may still include the possibility that the treatment with nanoplastics during pregnancy could have had an indirect effect on embryo development by altering the physiological state of the mother.

The female-specific cognitive impairment caused by PSNPs observed in this study may come from the sexual dimorphism of the developing brain. In this regard, gender-specific synaptic development, functioning, and neurotransmitter activity in the hippocampus have all been reported ${ }^{30}$. In particular, the 
287 level of estrogen receptor $\alpha(E R \alpha)$ is higher in the hippocampus of female mice than in that of male 288 mice, and ERa signaling is a key mediator of the induction of long-term potentiation in female mice ${ }^{31,32}$. 289 Consistent with these reports, we observed that PSNP exposure decreased the expression of ERa in 290 the hippocampus of female progeny (unpublished data; data not shown). Further study is required to 291 investigate the molecular mechanisms underlying PSNP-induced brain developmental abnormalities. 


\section{PSNPs used in the study}

295

296

297

298

299

300

301

302

303

304

305

306

307

308

309

310

311

312

313

314

315

316

317

PSNPs used in the current study are Yellow-Green (YG)-conjugated particles with an excitation and emission wavelengths of 441 and $486 \mathrm{~nm}$, respectively (Polyscience, Warrington, PA). A couple of different sizes (50 nm and $500 \mathrm{~nm}$ ) of plain and carboxylated PSNPs (2.5\% w/v aqueous suspensions) were evaluated for bioaccumulation and neurological effects. Carboxylated NPs have negatively charged carboxylate groups ( $\mathrm{COOH}-)$ on their surfaces. Plain PSNPs have no surface modification but are negative charge stabilized colloidal particles. Aqueous suspension $(2.5 \% \mathrm{w} / \mathrm{v})$ of $50 \mathrm{~nm}$ PSNPs and $500 \mathrm{~nm}$ PSNPs include $3.64 \times 10^{14}$ particles $/ \mathrm{ml}$ and $3.64 \times 10^{11}$ particles $/ \mathrm{ml}$, respectively.

\section{Mice}

Pregnant female C57BL/6J mice were purchased from Daehan Biolink Co. (Korea). Mice used in the experiment were fed AIN-93G diet to minimize autofluorescence and enhance the accuracy of tissue analysis. All animal experiments complied National Institutes of Health Guidelines and were approved by Animal Use and Care Committee protocol at Korea Research Institute Bioscience \& Biotechnology (KRIBB) (Permit number: KRIBB-AEC-19193).

\section{Treatment of PSNPs in vivo}

For oral administration of PSNPs to the pregnant and lactating mother, PSNP (carboxylated; $50 \mathrm{~nm}$ of diameter) containing agarose jelly cubes (0-1,000 $\mu \mathrm{g}$ PSNP, $0.1 \mathrm{~g}$ nut powder, $10 \%$ sucrose, $2.5 \%$ agarose $/ \mathrm{cm}^{3}$ of each cube) were prepared (Supplementary figure 2b). PSNP containing jelly cubes were treated once a day from the $8^{\text {th }}$ day of pregnancy. A group of mice treated with agarose jelly cubes containing nuts and sucrose but no PSNP was used as control.

\section{Immunofluorescence staining}


Brain tissues and neurospheres were cryosectioned and immunolabeled as previously described 33,34 .

319 Primary antibodies used in the experiments were: anti-NeuN (1:100; MilliporeSigma; Burlington, MA), 320 anti-Neurofilament (1:500; Abcam, Cambridge, UK), anti-Ki67 (1:100; BD Biosciences, San Jose, CA), 321 anti-Nestin (1:500; Abcam) and anti-GFAP (1:200; Thermo Fisher Scientific, Waltham, MA) antibodies.

322 The signals were visualized with appropriate fluorescence conjugated secondary antibodies. Hoechst 32333258 was used for counterstaining (Life technologies, Carlsbad, CA). The images were obtained with 324 fluorescence microscope (Zeiss, Germany) and confocal microscope (Zeiss, Germany).

\section{H\&E staining}

327 For histological analysis, various mouse tissues were stained with hematoxylin for nuclear staining followed by the staining with eosin to visualize cytoplasmic regions. The images were obtained using light microscope (Olympus, Japan).

\section{Culture of mouse hippocampal NSCs}

Hippocampal regions were dissected from embryonic (E16.5) mouse brain. Primary hippocampal NSCs were prepared as described previously ${ }^{33,34}$.

\section{Treatment of PSNPs in vitro}

Biological effects of PSNPs on the functions of primary hippocampal NSCs were examined with two different sizes (50 nm and $500 \mathrm{~nm}$ ) of carboxylated and plain PSNPs at $0-100 \mu \mathrm{g} / \mathrm{ml}$. No PSNP containing distilled water was treated to the control group.

\section{NSC proliferation assay}

341 Neurospheres were dissociated into single cells using $0.1 \%$ Trypsin-EDTA solution. Dissociated cells 
342 (3,000 cells/well) were seeded into 24-well ultra-low attachment plates with NSC growth medium. After

$343 \quad 7-9$ days the proliferation rate was measured as previously described 33,34 .

\section{Multi-lineage differentiation assay}

346 Neurospheres treated with vehicle and PSNPs $(25 \mu \mathrm{g} / \mathrm{ml})$ were dissociated into single NSCs followed 347 by plating onto matrigel coated-24 well culture plate $\left(1.5 \times 10^{5}\right.$ cells/well $)$ with differentiation medium 348 (DMEM/F12 media, N2, B27, Glutamax) to induce neuronal and glial differentiation. After 7-9 days, the cells were immunolabeled with anti-Tuj1 (1:1,000; Biolegend) and anti-GFAP (1:200; Thermo Fisher Scientific) antibodies for neurons and astrocytes, respectively ${ }^{33,34}$.

\section{Transcriptome and network analysis}

For transcriptome analysis, RNA was prepared from mouse embryonic NSCs (E16.5) treated with 25 $\mu \mathrm{g} / \mathrm{ml}$ carboxylated PSNPs (50 $\mathrm{nm}$ of diameter). mRNA-seq library construction was performed with MGIEasy RNA Directional Library Prep Kit (MGISEQ) according to the manufacturer's instructions. Adapter and low quality of FASTQ reads were trimmed using Trim Galore with option "-p 20". After trimming, differential gene expression analysis of mRNA-seq experiment was carried out on Tuxedo protocol $^{35}$. Briefly, trimmed FASTQ reads were mapped to GENCODE's mm10 genome (GRCm38.p6) using Tophat ${ }^{36}$ with options "-p 16 -r 250 -mate-std-dev 50 -library-type fr-secondstrand -G /path/ gencode.vM24.annotation.gtf". Because Tophat considers splicing junctions between exons, we used GTF file from GENCODE (gencode.vM24.annotation.gtf). To quantify gene and transcript expression, we used Cuffquant with options "-frag-bias-correct -multi-read-correct -num-threads 16 -library-type frsecondstrand -M/path/ gencode.vM24.annotation.mask.gtf". In order to compute the gene and transcript expression more effectively, we used "-M mask file" options for ignoring all alignment within transcript in mask file. Mask file was made by linux command "grep -v 'protein coding' gencode.vM24.annotation.gtf > gencode.vM24.annotation.mask.gtf". This mask file doesn't have protein coding genes, miRNAs, and IncRNAs. We normalized the expression levels using Cuffnorm with options "-num-threads 16 -library-type fr-secondstrand -library-norm-method classic-fpkm -output- 
format simple-table". To compare expression levels between control and C50nm, we used Cuffdiff with options "-frag-bias-correct -multi-read-correct -num-threads 16 -library-type fr-secondstrand -librarynorm-method classic-fpkm -dispersion-method blind". We set differentially expressed gene criteria as "fold change of FPKM is more than 1.5 and p-value of Cuffdiff is less than 0.05 ". We called these genes differentially expressed genes (DEGs).

\section{Gene Ontology}

Gene ontology analysis was performed by Metascape with DEGs ${ }^{37}$. We used GO biological process, KEGG pathways, Reatome gene sets, CORUM complexes, and canonical pathways from MSigDB by default. Analysis parameters were used with enriched terms to include $\geq 3$ candidates, $p$-value $\leq 0.01$, and enrichment factor $\geq 1.5$.

\section{Protein-Protein Interaction (PPI) Network Analysis}

Network analysis was performed by NetworkAnalyst ${ }^{38}$. We analyzed PPI based on STRING interactome database. At first, we used basic options with confidence score cutoff 900 to find out how many down-regulated DEGs are connected closely. To identify direct interaction with DEGs, we used "zero-order Network" option that shows directly connected gene.

\section{Electrophysiology}

PSNP (500 $\mu \mathrm{g} / \mathrm{day}$; carboxylated PSNP; $50 \mathrm{~nm}$ of diameter) treated mice at the age of 10-12 weeks were used for electrophysiological experiment. All animals were anesthetized by using isoflurane, and theirs brain were stored in cold choline dissection buffer (containing in mM: 110 choline chloride, 11.6 Na-ascorbate, 3.1 pyruvate, $25 \mathrm{NaHCO}_{3}, 1.25 \mathrm{NaH}_{2} \mathrm{PO}_{4}, 2.5 \mathrm{KCl}, 7 \mathrm{MgCl}_{2}, 0.5 \mathrm{CaCl}_{2}$ and 25 glucose, bubbled with $95 \% \mathrm{O}_{2}$ and $5 \% \mathrm{CO}_{2}$ ). Coronal brain slices containing the hippocampus (300 $\mathrm{mm}$ thick) were prepared using a Leica VT1000S vibrating tissue slicer and placed in a submerged chamber with an artificial cerebrospinal fluid (aCSF, containing in mM: $1 \mathrm{NaH}_{2} \mathrm{PO}_{4}, 26.2 \mathrm{NaHCO}_{3}, 118 \mathrm{NaCl}, 2.5 \mathrm{KCl}$, 
11 glucose, $2 \mathrm{CaCl}_{2}$ and $1 \mathrm{MgCl}_{2}$, bubbling with $95 \% \mathrm{O}_{2}$ and $5 \% \mathrm{CO}_{2}$ ) at $35^{\circ} \mathrm{C}$ water bath for $45 \mathrm{~min}$. Brain slices were kept at room temperature before recording and transferred to the recording chamber perfusing aCSF maintained at $29-32^{\circ} \mathrm{C}$. Field excitatory postsynaptic potentials (fEPSP) were recorded using a DAM-80 amplifier and WIN LTP 2.10 software (University of Bristol, UK), filtered $3 \mathrm{kHz}$ and sampled at $20 \mathrm{kHz}$. Recording pipettes with resistance of 1-3M $\Omega$ were filled with aCSF. fEPSPs were obtained from CA1 dendritic areas by stimulating stratum radiatum alternatively with 20 s interstimulation intervals by using two bipolar electric stimulators (FHC Inc, ME, USA). LTP was induced by giving 4 times of theta burst stimulation (4xTBS: a single TBS is composed of TBS, 20 bursts of 4 pulses at $100 \mathrm{~Hz}$, repeated 4 times in 10s intervals). Low frequency stimulation (LFS: 600 pulses of $1 \mathrm{~Hz}$ ) was used to induce $\operatorname{LTD}^{39,40}$.

\section{Measurement of glutamate/GABA in the cortex and the hippocampus}

For the analysis of neurotransmitters, brain tissues were isolated from 11 weeks old control and PSNP (500 $\mu \mathrm{g} /$ day; carboxylated PSNP; $50 \mathrm{~nm}$ of diameter) treated mice. The levels of glutamate and GABA were determined by high performance liquid chromatography (HPLC, 1260 Infinity system, Agilent Technologies, Santa Clara, CA) using an UV detector ${ }^{41,42}$. Briefly, the brain tissues were homogenized in distilled water and centrifuged, and the supernatants derivatized with o-phthaldialdehyde were injected using an autosampler at $4^{\circ} \mathrm{C}$ followed by the elution through an Eclipse Plus RR-C18 column $(4.6 \times 100 \mathrm{~mm} \times 3.5 \mu \mathrm{m}$, Agilent Technologies, Santa Clara, CA $)$ at $45 \pm 1^{\circ} \mathrm{C}$ with a mobile phase. The composition of the mobile phase $\mathrm{A}$ was $40 \mathrm{mM} \mathrm{Na}_{2} \mathrm{HPO}_{4}$ and $0.1 \%$ phosphoric acid, and mobile phase B was acetonitrile/methanol/water (45/45/10, v/v/v). Compounds were eluted over a 17 min runtime at a flowrate of $1 \mathrm{ml} / \mathrm{min}$ with gradient conditions (linear gradient change from $5-50 \%$ of mobile phase $B$ between 0 and $15 \mathrm{~min} ; 15-16 \mathrm{~min}$, linear gradient change from 50 - 80\% mobile phase $\mathrm{B} ; 16-17 \mathrm{~min}$, linear gradient change from $80 \%$ back to $5 \%$ mobile phase B). The peaks of glutamate/GABA were analyzed and integrated using a ChemStation software (Agilent Technologies, Santa Clara, CA), and all samples were normalized based on the protein concentration spectrophotometrically determined by BCA protein assay (Thermo Scientific, Waltham, MA). 
424 All the behavior tests were performed according to the time line illustrated in supplementary figure 425 1. PSNPs (500 $\mu \mathrm{g} /$ day; carboxylated PSNP; $50 \mathrm{~nm}$ of diameter) were maternally administered during 426 pregnant and lactating periods (from embryonic day 8 until 2 weeks after birth) and the tests were 427 performed in the progeny at 8-10 weeks after birth.

- Y-maze alternation test: The Y-maze alternation test was performed as previously described ${ }^{43,44}$. A Ymaze with three identical arms of plexiglass $(51.5 \times 11.5 \times 12 \mathrm{~cm})$, which were $120^{\circ}$ apart, was used. Each mouse was placed in a Y-maze and allowed to explore the maze for $10 \mathrm{~min}$. The sessions were video recorded and scored for entries into arms. The percentage of spontaneous alternation was calculated as the ratio of the actual to possible alternations (defined as the total number of arm entries minus 2), which was multiplied by 100 : alternation $(\%)=$ [(number of alternation) $/$ (total arm entries-2)] $x 100$.

- Novel object recognition test (NOT): The NOT was conducted as previously described ${ }^{44}$. Mice were individually habituated to a testing chamber $(40 \mathrm{~cm}$ wide $\times 20$ long $\times 20 \mathrm{~cm}$ high) with no objects for 5 min. Mice were placed in a testing chamber for 10 min with two identical objects (familiar objects). 24 hr later mice were placed into the testing chamber in the presence of one of the familiar objects and one novel object (novel object) for $10 \mathrm{~min}$. The familiar objects were cylindrical wooden blocks $10 \mathrm{~cm}$ high $\times 2 \mathrm{~cm}$ diameter. The novel object was a $10 \mathrm{~cm} \times 2.5 \mathrm{~cm} \times 2 \mathrm{~cm}$ rectangular wooden block. The acquisition and recognition sessions were video-recorded and scored the time spent exploring the objects. The chambers and objects were cleaned with ethanol between trials. Exploration was defined as sniffing and touching the object with the nose and/or forepaws. Sitting on the object was not considered exploratory behaviour. A discrimination index was calculated for each animal and expressed using the following formula: (exploring the novel object - exploring the familiar object)/ (exploring the novel object + exploring the familiar object) on day 2 .

447 - Open field test: The open field test was conducted as previously described ${ }^{45}$. Animals were habituated in the test room for 30 min before the initial testing. Mice were individually placed in an open field box $\left(45 \times 45 \times 45 \mathrm{~cm}^{3}\right)$ for $30 \mathrm{~min}$. The horizontal locomotion of the mouse was measured using a computerized video tracking system, SMART (Panlab, Barcelona, Spain). 
451 - Social interaction test: Each mouse was placed in the white acryl wall box $(40 \times 20 \times 20 \mathrm{~cm})$ for 3 min

452 for habituation. After then, an age matched novel C57BL/6J control male mouse was introduced to the 453 test cage and allowed to explore freely for $3 \mathrm{~min}$. The sessions were video recorded and social 454 interaction, such as body sniffing, anogenital sniffing, and direct contact was analyzed for 3 min.

455

456 Statistical analysis of the data

457 All data were obtained from at least three replicates and analyzed by unpaired t-test or one-way 458 ANOVA test (Newman-Keuls method). Statistical analyses were performed with GraphPad Prism 459 (version 5.0, GraphPad Software, USA), and $p$ values less than 0.05 interpreted as significant 460 differences.

461

462 Data availability

463 The authors declare that the data supporting the findings of this study are available within the paper 464 and its supplementary information files. 
4671 Shim, W. J. \& Thomposon, R. C. Microplastics in the Ocean. Arch Environ Contam Toxicol 69, 468 265-268, doi:10.1007/s00244-015-0216-x (2015).

4692 Andrady, A. L. Microplastics in the marine environment. Mar Pollut Bull 62, 1596-1605, 470 doi:10.1016/j.marpolbul.2011.05.030 (2011).

4713 Napper, I. E., Bakir, A., Rowland, S. J. \& Thompson, R. C. Characterisation, quantity and 472 sorptive properties of microplastics extracted from cosmetics. Mar Pollut Bull 99, 178-185, 473 doi:10.1016/j.marpolbul.2015.07.029 (2015).

4744 Lehner, R., Weder, C., Petri-Fink, A. \& Rothen-Rutishauser, B. Emergence of Nanoplastic in 475 the Environment and Possible Impact on Human Health. Environ Sci Technol 53, 1748-1765, 476 doi:10.1021/acs.est.8b05512 (2019).

$4775 \quad$ Guterres, S. S., Alves, M. P. \& Pohlmann, A. R. Polymeric nanoparticles, nanospheres and 478 nanocapsules, for cutaneous applications. Drug Target Insights 2, 147-157 (2007).

4796 Wesch, C., Bredimus, K., Paulus, M. \& Klein, R. Towards the suitable monitoring of ingestion 480 of microplastics by marine biota: A review. Environ Pollut 218, 1200-1208, 481 doi:10.1016/j.envpol.2016.08.076 (2016).

4827 Lee, W. S. et al. Bioaccumulation of polystyrene nanoplastics and their effect on the toxicity of 483 Au ions in zebrafish embryos. Nanoscale 11, 3173-3185, doi:10.1039/c8nr09321k (2019).

4848 Pitt, J. A. et al. Uptake, tissue distribution, and toxicity of polystyrene nanoparticles in 485 developing zebrafish (Danio rerio). Aquat Toxicol 194, 185-194, 486 doi:10.1016/j.aquatox.2017.11.017 (2018).

4879 Moller, J. N., Loder, M. G. J. \& Laforsch, C. Finding Microplastics in Soils: A Review of Analytical 488 Methods. Environ Sci Technol 54, 2078-2090, doi:10.1021/acs.est.9b04618 (2020).

48910 Zhang, Y. et al. Atmospheric microplastics: A review on current status and perspectives. Earth$490 \quad$ Science Reviews 203, 103118 (2020).

49111 Cox, K. D. et al. Human Consumption of Microplastics. Environ Sci Technol 53, 7068-7074, 492 doi:10.1021/acs.est.9b01517 (2019).

49312 Deng, Y., Zhang, Y., Lemos, B. \& Ren, H. Tissue accumulation of microplastics in mice and 494 biomarker responses suggest widespread health risks of exposure. Sci Rep 7, 46687, 
doi:10.1038/srep46687 (2017).

3 Jin, H. et al. Polystyrene microplastics induced male reproductive toxicity in mice. Journal of Hazardous Materials 401, 123430 (2020).

4 Hwang, J. et al. Potential toxicity of polystyrene microplastic particles. Scientific reports 10, 1$12(2020)$.

15 Keelan, J. A. Nanotoxicology: nanoparticles versus the placenta. Nature nanotechnology 6, 263 (2011).

6 Saliu, F. et al. Microplastic and charred microplastic in the Faafu Atoll, Maldives. Marine pollution bulletin 136, 464-471 (2018).

17 Harrison, J. P., Ojeda, J. J. \& Romero-González, M. E. The applicability of reflectance microFourier-transform infrared spectroscopy for the detection of synthetic microplastics in marine sediments. Science of the Total Environment 416, 455-463 (2012).

18 Bennett, D. et al. Project TENDR: Targeting Environmental Neuro-Developmental Risks The TENDR Consensus Statement. Environ Health Perspect 124, A118-122, doi:10.1289/EHP358 (2016).

Cressman, A. M. et al. Maternal cocaine use during breastfeeding. Can Fam Physician 58, 1218-1219 (2012).

20 Saunders, N. R., Dziegielewska, K. M., Mollgard, K. \& Habgood, M. D. Physiology and molecular biology of barrier mechanisms in the fetal and neonatal brain. $J$ Physiol 596, 57235756, doi:10.1113/JP275376 (2018).

21 Chen, Q. et al. Quantitative investigation of the mechanisms of microplastics and nanoplastics toward zebrafish larvae locomotor activity. Science of the total environment 584, 1022-1031 (2017).

22 da Costa Araújo, A. P. \& Malafaia, G. Microplastic ingestion induces behavioral disorders in mice: A preliminary study on the trophic transfer effects via tadpoles and fish. Journal of Hazardous Materials 401, 123263 (2020).

23 Ragusa, A. et al. Plasticenta: Microplastics in Human Placenta. bioRxiv (2020).

24 Schwertfeger, K. L., McManaman, J. L., Palmer, C. A., Neville, M. C. \& Anderson, S. M. Expression of constitutively activated Akt in the mammary gland leads to excess lipid synthesis 

(2003).

52625 Huang, F. L. \& Huang, K. P. Methylphenidate improves the behavioral and cognitive deficits of neurogranin knockout mice. Genes Brain Behav 11, 794-805, doi:10.1111/j.1601183X.2012.00825.x (2012).

26 Bethel-Brown, C., Yao, H., Hu, G. \& Buch, S. Platelet-derived growth factor (PDGF)-BBmediated induction of monocyte chemoattractant protein 1 in human astrocytes: implications for HIV-associated neuroinflammation. J Neuroinflammation 9, 262, doi:10.1186/1742-2094-9262 (2012).

Lafuente, J. V. et al. Expression of vascular endothelial growth factor (VEGF) and plateletderived growth factor receptor-beta (PDGFR-beta) in human gliomas. J Mol Neurosci 13, 177185, doi:10.1385/JMN:13:1-2:177 (1999).

S. et al. Transfer studies of polystyrene nanoparticles in the ex vivo human placenta perfusion model: key sources of artifacts. Sci Technol Adv Mater 16, 044602, doi:10.1088/1468-6996/16/4/044602 (2015). Yamashita, K. et al. Silica and titanium dioxide nanoparticles cause pregnancy complications in mice. Nature nanotechnology 6, 321-328 (2011).

$54833 \mathrm{Kim}, \mathrm{T}$. H. et al. Copine1 regulates neural stem cell functions during brain development. Biochem Biophys Res Commun 495, 168-173, doi:10.1016/j.bbrc.2017.10.167 (2018).

$55034 \quad$ Jeong, B. et al. Spastin Contributes to Neural Development through the Regulation of Microtubule Dynamics in the Primary Cilia of Neural Stem Cells. Neuroscience 411, 76-85, doi:10.1016/j.neuroscience.2019.05.024 (2019). 
$553 \quad 35$ Trapnell, C. et al. Differential gene and transcript expression analysis of RNA-seq experiments 554 with TopHat and Cufflinks. Nat Protoc 7, 562-578, doi:10.1038/nprot.2012.016 (2012).

$55536 \mathrm{Kim}$, D. et al. TopHat2: accurate alignment of transcriptomes in the presence of insertions, 556 deletions and gene fusions. Genome Biol 14, R36, doi:10.1186/gb-2013-14-4-r36 (2013).

55737 Zhou, Y. et al. Metascape provides a biologist-oriented resource for the analysis of systems558 level datasets. Nat Commun 10, 1523, doi:10.1038/s41467-019-09234-6 (2019).

55938 Zhou, G. et al. NetworkAnalyst 3.0: a visual analytics platform for comprehensive gene 560 expression profiling and meta-analysis. Nucleic Acids Res 47, W234-W241, doi:10.1093/nar/gkz240 (2019).

56239 Park, H., Rhee, J., Lee, S. \& Chung, C. Selectively Impaired Endocannabinoid-Dependent Long-Term Depression in the Lateral Habenula in an Animal Model of Depression. Cell Rep 20, 289-296, doi:10.1016/j.celrep.2017.06.049 (2017). Rhee, J., Park, K., Kim, K. C., Shin, C. Y. \& Chung, C. Impaired Hippocampal Synaptic Plasticity and Enhanced Excitatory Transmission in a Novel Animal Model of Autism Spectrum Disorders with Telomerase Reverse Transcriptase Overexpression. Mol Cells 41, 486-494, doi:10.14348/molcells.2018.0145 (2018). Monge-Acuna, A. A. \& Fornaguera-Trias, J. A high performance liquid chromatography method with electrochemical detection of gamma-aminobutyric acid, glutamate and glutamine in rat brain homogenates. J Neurosci Methods 183, 176-181, doi:10.1016/j.jneumeth.2009.06.042 (2009). chromatographic method for GABA and glutamate determination in regions of the rodent brain. J Neurosci Methods 177, 289-293, doi:10.1016/j.jneumeth.2008.10.011 (2009). Belforte, J. E. et al. Postnatal NMDA receptor ablation in corticolimbic interneurons confers schizophrenia-like phenotypes. Nature neuroscience 13, 76-83, doi:10.1038/nn.2447 (2010). (2017).

Park, T. S. et al. Humulus japonicus inhibits the progression of Alzheimer's disease in a APP/PS1 transgenic mouse model. Int J Mol Med 39, 21-30, doi:10.3892/ijmm.2016.2804

Go, J. et al. Piperlongumine decreases cognitive impairment and improves hippocampal 
583

584 
586 This study was supported by the grants in below:

587 - The KRIBB Initiative Research Program, grant number: KGM5222012

588 - The Brain Research Program of the National Research Foundation (NRF) funded by the Korean 589 government (MSIT), grant numbers: NRF-2019M3C7A1031534 (D.Y.L.), NRF5902015 M3C7A1029113 (J.-R.L.), and NRF-2019M3C7A1031742 (C.C.)

591 - Basic Science Research Program of the NRF funded by Korean government (Ministry of 592 Education), grant number: NRF-2019R1I1A2A01063642 (D.Y.L.)

593 - The National Research Foundation of Korea (NRF) grant funded by the Korea government (MSIT), 594 grant number: NRF-2019R1A2C2006740 (T.-K.K.), NRF- 2019R1A5A6099645 (T.-K.K.)

595 - The Basic Science Research Program through the National Research Foundation of Korea (NRF) 596 funded by Korea government (MSIT), grant number: NRF-2019R1C1C1006084 (J.J.)

\section{Author information}

B.J. and D.Y.L. conceived the project, designed the experiments and wrote the paper. B.J. carried out the treatment of PSNP into mice, histological analysis and in vitro experiments. Y.-K.R. and K.-S.K performed the behavior tests of mice and analyzed the data. J.K., S.P. performed mouse tissue sampling and histological analysis. J.Y.B. and W.-H.S analyzed HPLC data and performed statistical analysis. S.Z. and C.C performed ex vivo electrophysiological recordings and analyzed the data. R.D., H-S.C., and T.-K.K. performed mRNA-seq experiments and D.U. and T.-K.K. analyzed the transcriptome data and protein-protein interaction network. W.S.L. and J.J. performed nanoparticle characterization. J.-R.L., N.-S.K and D.Y.L. supervised the project and all authors contributed to the discussion for carrying out the project.

\section{Ethics declarations}

The authors declare that they have no competing interests. 
612 Corresponding author

613 Correspondence to Da Yong Lee

614 
a

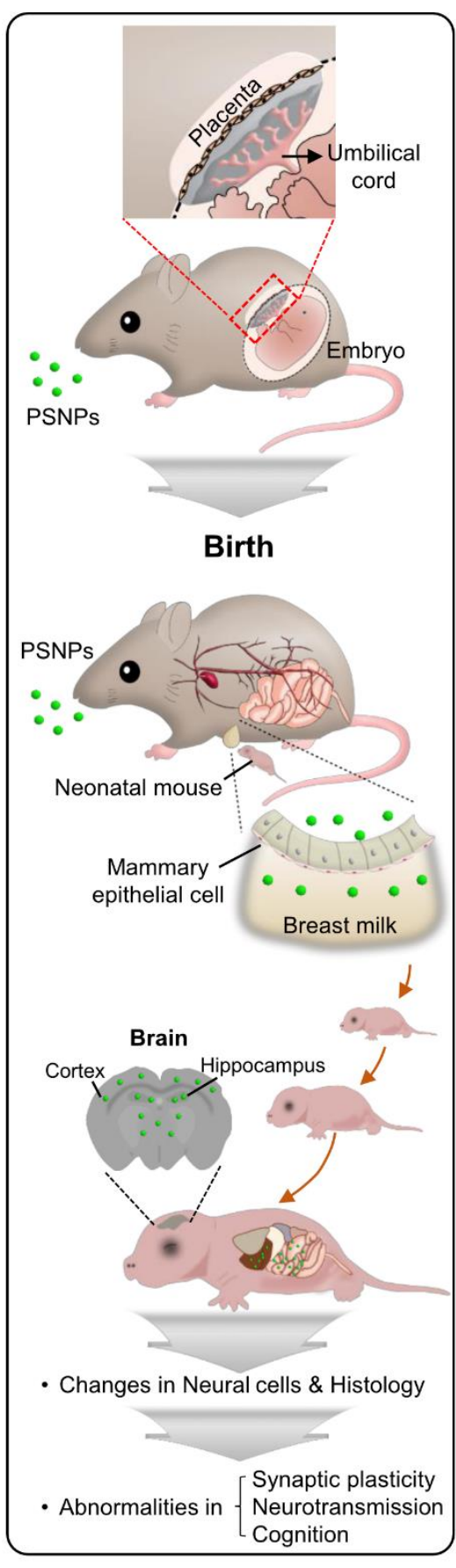

b
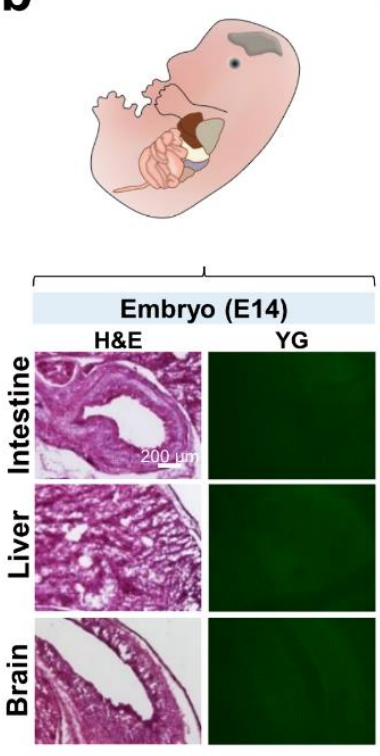

d

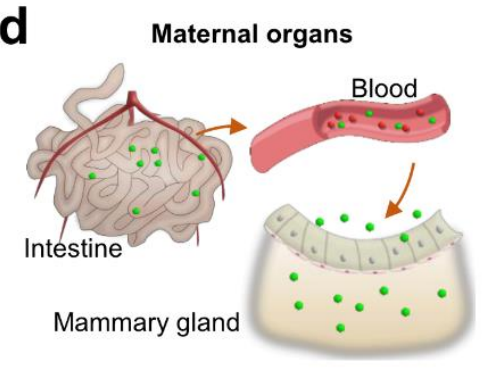

e

Offspring organs Intestine

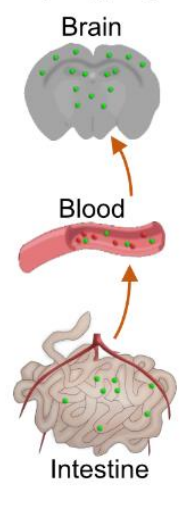

C

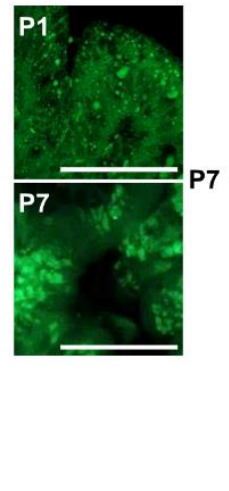


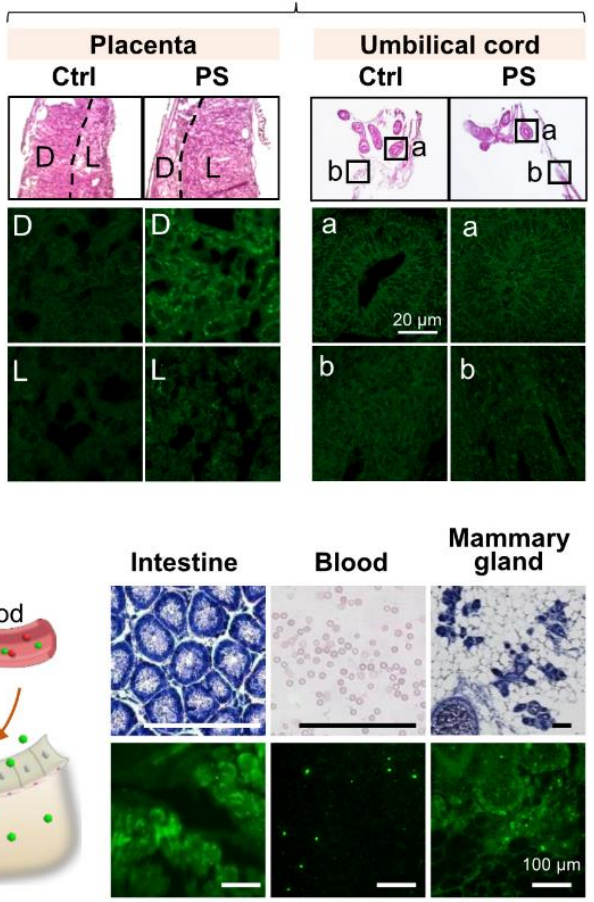

Brain

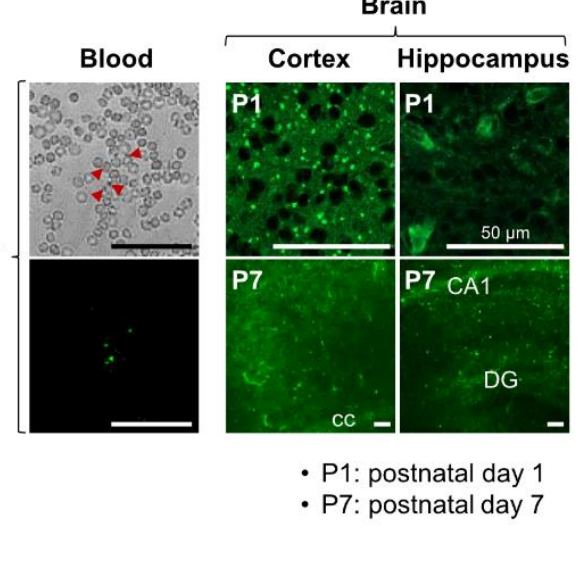


617 Figure 1. Maternally-administered PSNPs infiltrated various organs of the offspring and lactating

618 mother. (a) Schematic drawing shows the overall experimental procedures. (b) The tissue analysis 619 data show no YG fluorescence in embryonic tissues after maternal administration of PSNPs. (c) YG620 conjugated PSNPs were detected in the maternal side of the placenta (decidua; D), but very few were 621 detected in the fetal side of the placenta (labyrinth; L) after PSNP administration (PS). No fluorescence 622 signal was observed in the umbilical cord of either control group (Ctrl) or PSNP treated group (PS). 623 Histological structures were confirmed by H\&E staining. (d) Green fluorescence images show the 624 accumulation of YG-conjugated PSNPs in maternal tissues including blood, intestines, and the liver. (e) 625 YG-conjugated PSNPs were detected in the blood and organs of offspring at P1 and P7 including 626 intestine, brain cortex, and hippocampus (cc: corpus callosum; DG: dentate gyrus).

627 
a

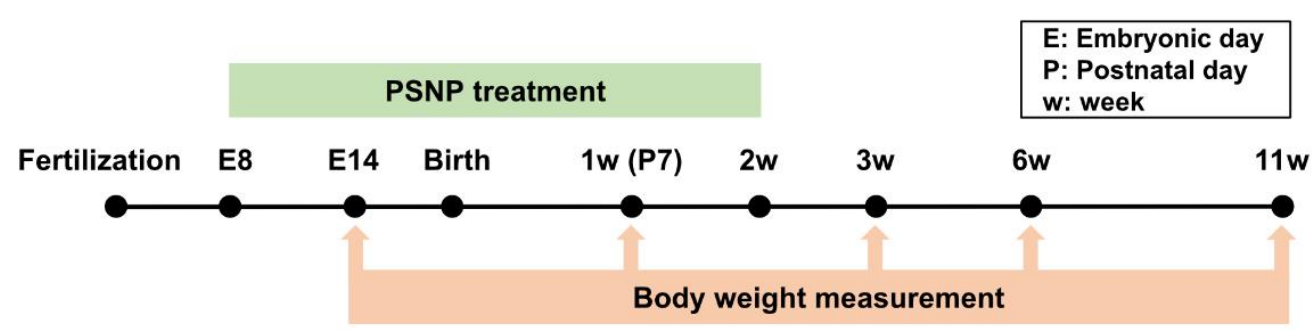

b
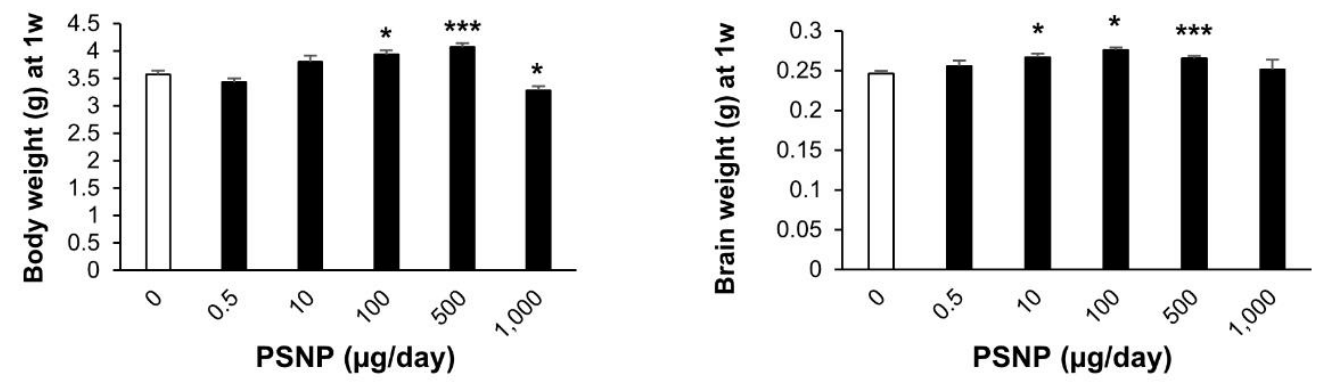

C
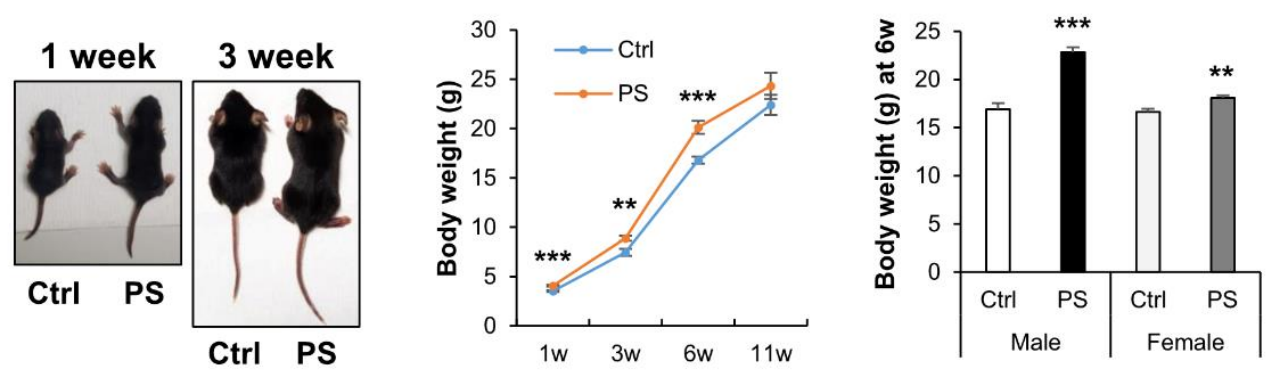

d
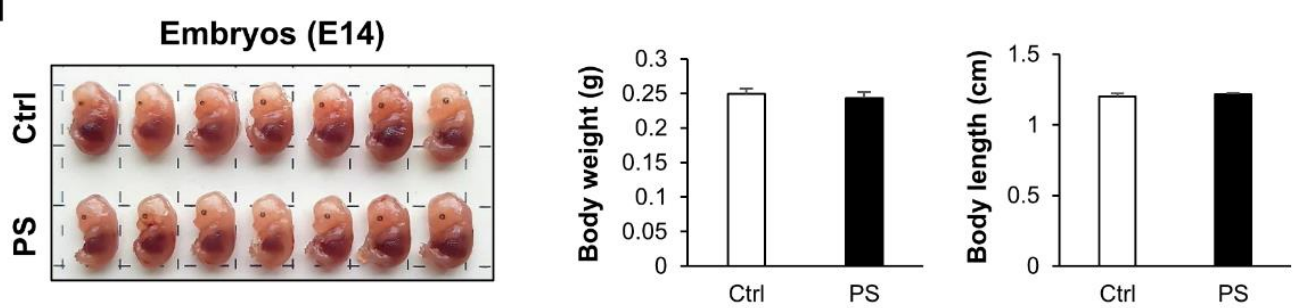
630 Figure 2. PSNPs increased the body and brain weights of the postnatal offspring. (a) Schematic

631 drawing shows various time points at which body and brain weights were measured. (b) The PSNP632 administered postnatal ( $1 \mathrm{w})$ group showed an increase in body weight and brain weight at doses of $633100-500 \mu \mathrm{g} /$ day. (c) The group that was administered $500 \mu \mathrm{g} /$ day of PSNPs (PS) showed the highest 634 body weight increase at 6 weeks $(6 \mathrm{w})$, regardless of gender, with the difference not being any more 635 significant at 11 weeks than in the controls (Ctrl). (d) No change in body weight or body length was 636 observed in E14 embryos after PSNP treatment (PS). Values denote mean \pm SEM. ${ }^{*} p<0.05,{ }^{* *} p<$ $6370.005,{ }^{* * *} p<0.0005$.

638 
a
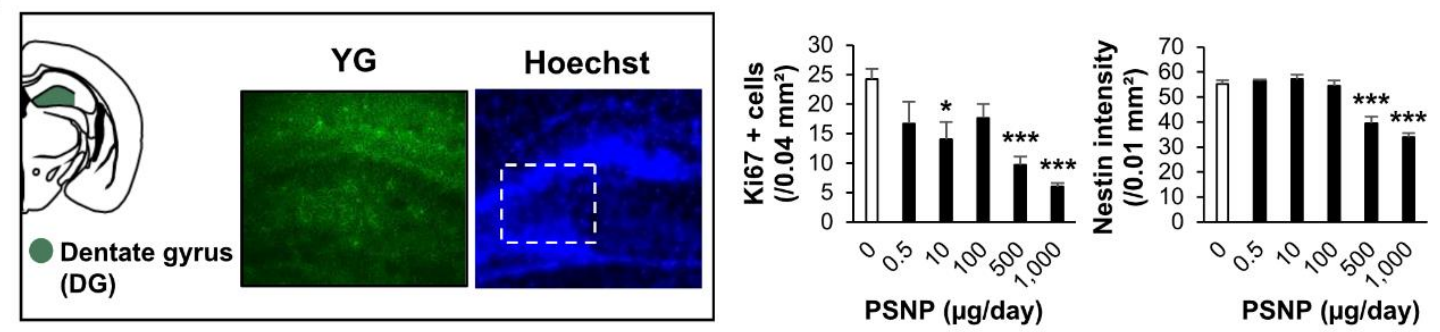

0

0.5

10

100

500

$1,000$ ( $\mu \mathrm{g} / \mathrm{day})$
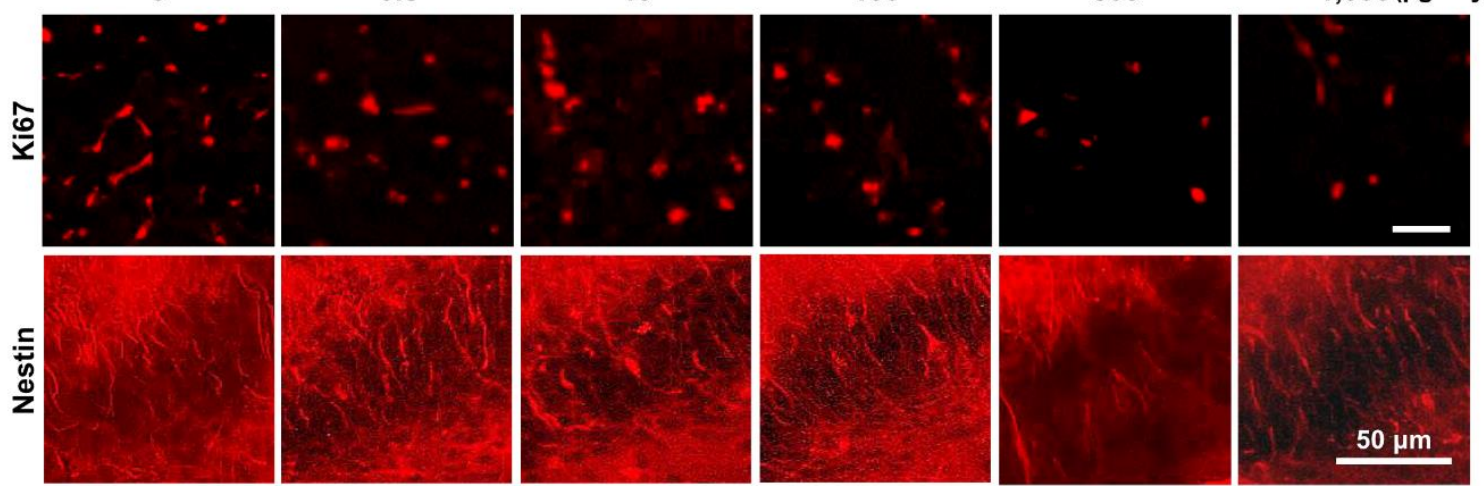

b

C

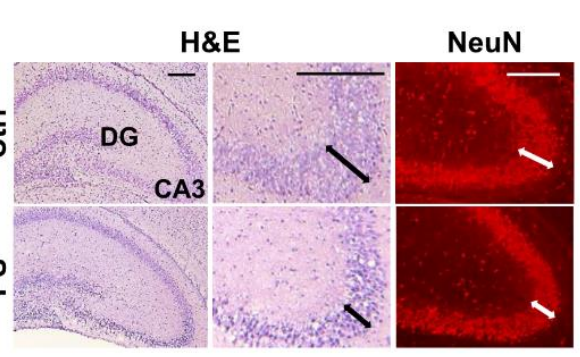

d NeuN Neurofilament H\&E
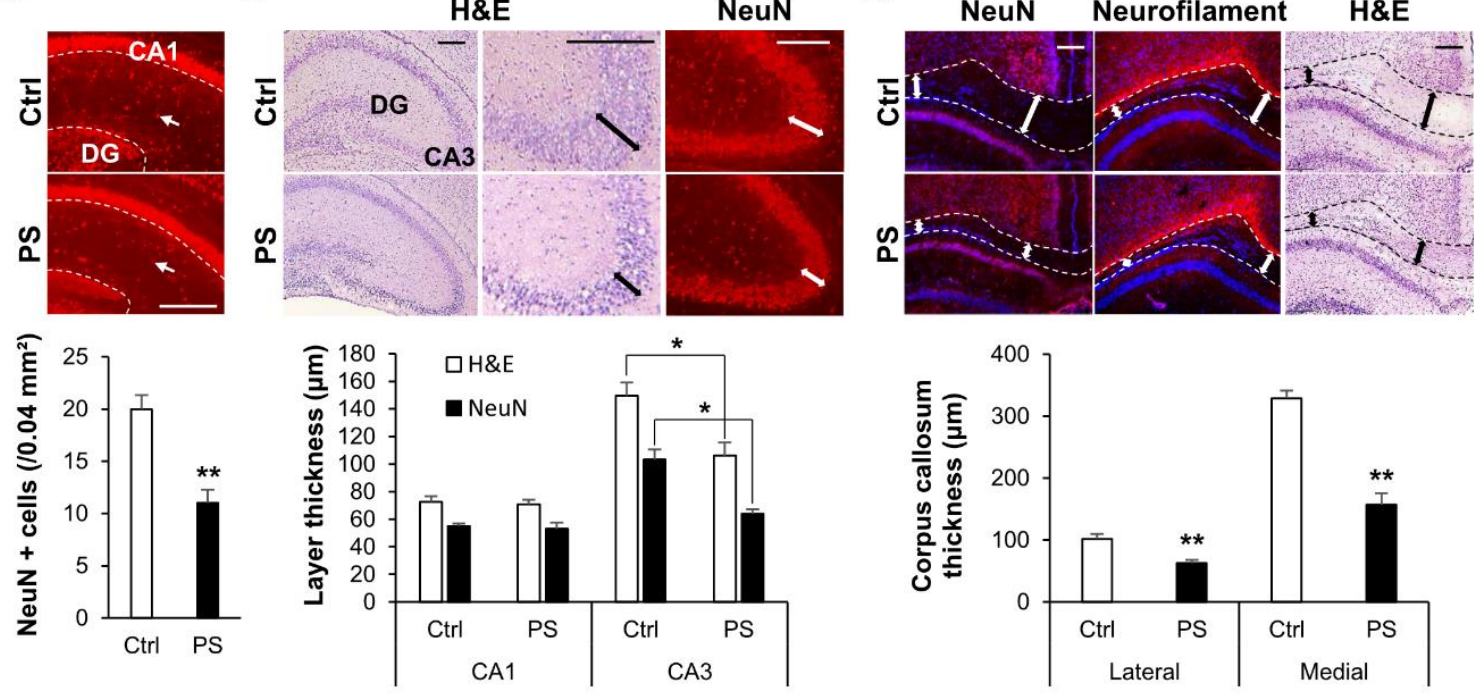

e

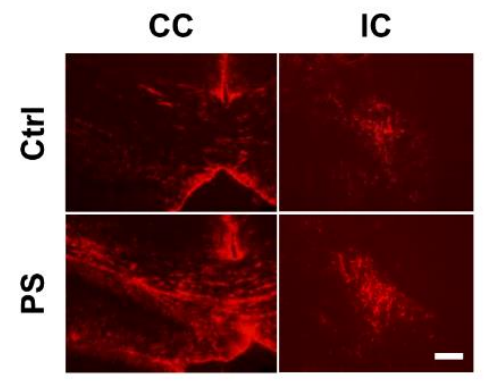


641 Figure 3. PSNPs altered neural cell composition in the brains of postnatal progeny. (a) The mouse

642 brain atlas shows the location of the hippocampal dentate gyrus (DG). A green fluorescence image

643 shows the accumulation of maternally-administered YG-conjugated carboxylated PSNPs (50-nm

644 diameter; $500 \mu \mathrm{g} /$ day) in the hippocampus of the progeny at P7. Immunofluorescence staining results

645 show that the number of Ki67+ and nestin+ progenitor cells was lower in the dentate gyrus of PSNP-

646 exposed groups (carboxylated; $50-\mathrm{nm}$ diameter) at $\geq 500 \mu \mathrm{g} /$ day. The number of Ki67+ cells and the

647 nestin signal intensity are shown graphically. (b) Immunofluorescence staining data show decreased

$648 \mathrm{NeuN}+$ neurons in the stratum radiatum and stratum lacunosum-moleculare of the hippocampus (arrow)

649 following PSNP administration (PS). (c) H\&E staining and NeuN immunolabeling data show reduced

650 thickness of the hippocampal CA3 layer following $500 \mu \mathrm{g} /$ day PSNP exposure. (d) Immunolabeling and

651 H\&E staining data show that the thickness of $\mathrm{NeuN}$ - and neurofilament+ corpus callosum was lower in

652 mice exposed to $500 \mu \mathrm{g} /$ day PSNP treatment. (e) The mouse brain atlas shows the structure of the

653 white matter including the corpus callosum (CC) and the internal capsule (IC). Immunofluorescence

654 staining data show that the GFAP+ signal intensity in the white matter (CC and IC) was higher in mice

655 exposed to $500 \mu \mathrm{g} /$ day of PSNPs. Values denote mean \pm SEM. ${ }^{*} p<0.05,{ }^{* *} p<0.005,{ }^{* * *} p<0.0005$.

656 Scale bars: (b, c) $200 \mu \mathrm{m},(\mathbf{d}, \mathbf{e}) 100 \mu \mathrm{m}$.

657 

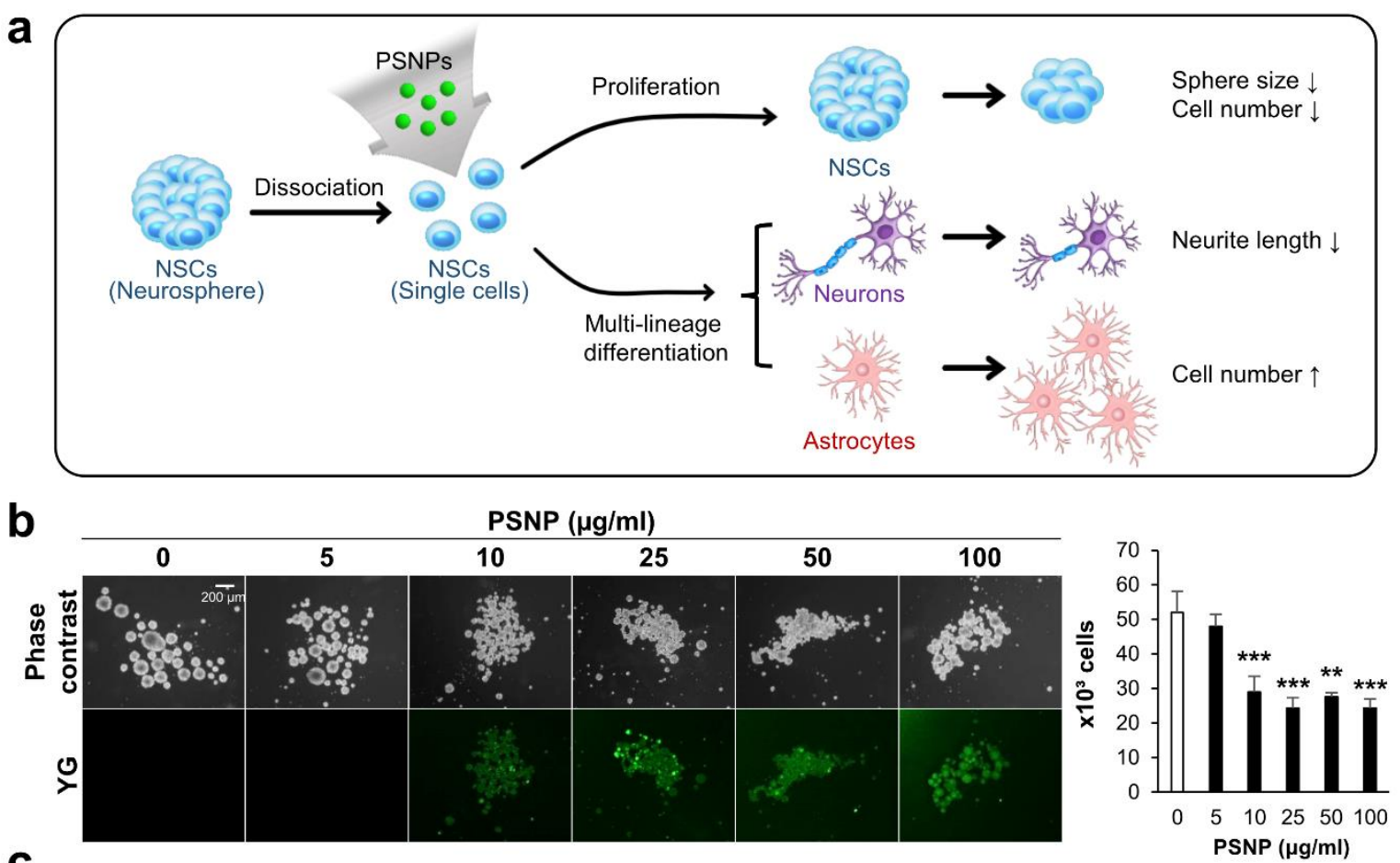

C
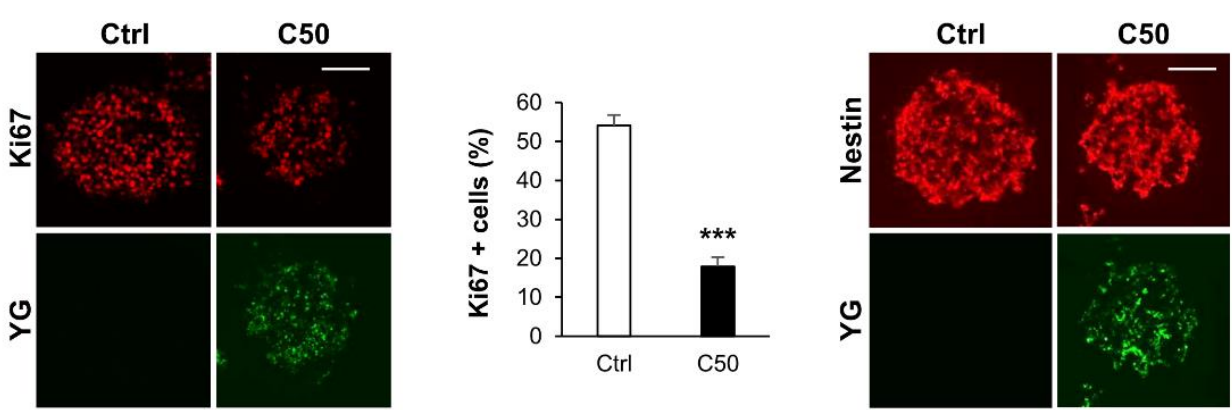

d

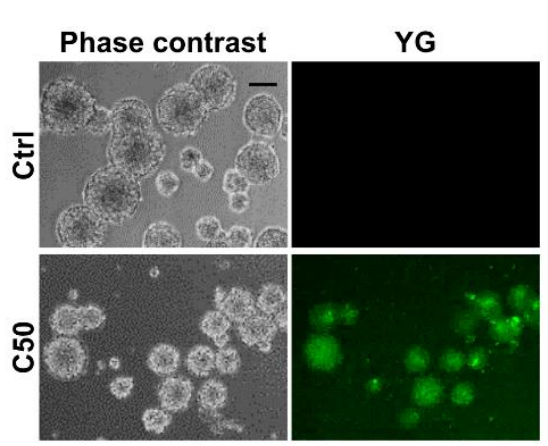

e
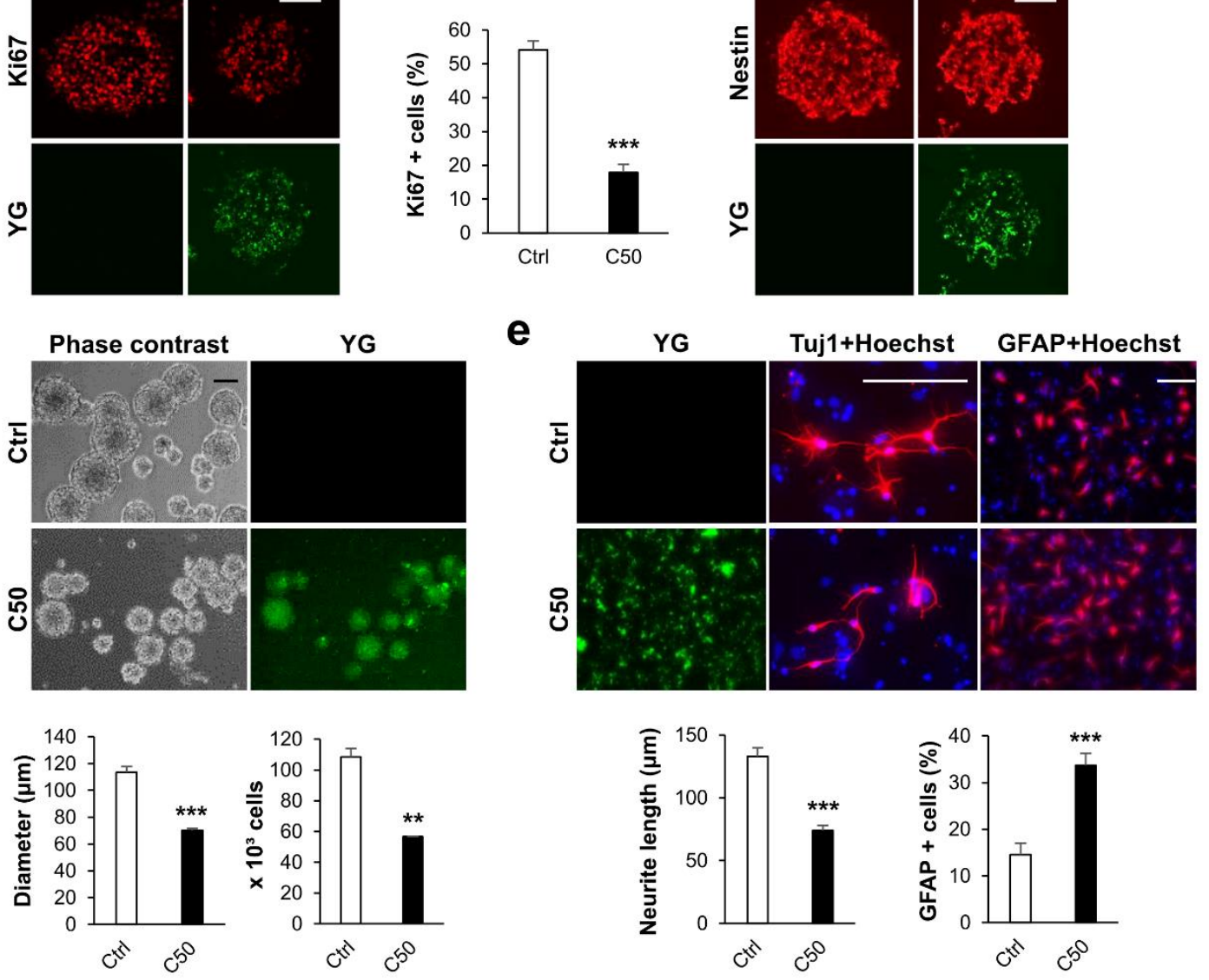
661 in vitro. (a) An illustration summarizing the in vitro procedure used to examine the effects of PSNPs on 662 NSC proliferation and multi-lineage differentiation. (b) The total cell number of NSCs was lower after exposure to $50 \mathrm{~nm}$ carboxylated PSNPs at $\geq 10 \mu \mathrm{g} / \mathrm{ml}$. (c) Immunofluorescence staining of single 664 neurospheres shows a decrease in Ki67+ proliferative NSCs following exposure to PSNPs (C50; 25 $\mu \mathrm{g} / \mathrm{ml}$ carboxylated PSNPs of $50 \mathrm{~nm}$ diameter). Other staining data show that nestin was well expressed

666 in the neurospheres of the control (Ctrl) and PSNP-exposed groups (C50). YG fluorescence signals 667 (green) were detected only in the PSNP-exposed group (C50). (d) Smaller neurosphere diameters and 668 total cell numbers were observed in the PSNP-exposed group than in the controls (Ctrl). (e) NSC differentiation assay data show a decreased neurite length of Tuj1+ neurons and a significant increase in GFAP+ astrocytes in the $25 \mu \mathrm{g} / \mathrm{ml}$ PSNP-treated group compared with controls. C: carboxylated PSNP; 50: $50 \mathrm{~nm}$. Values denote mean \pm SEM. ${ }^{* *} p<0.005,{ }^{* * *} p<0.0005$. Scale bars: $100 \mu \mathrm{m}$. 


\section{Downregulated genes}


b

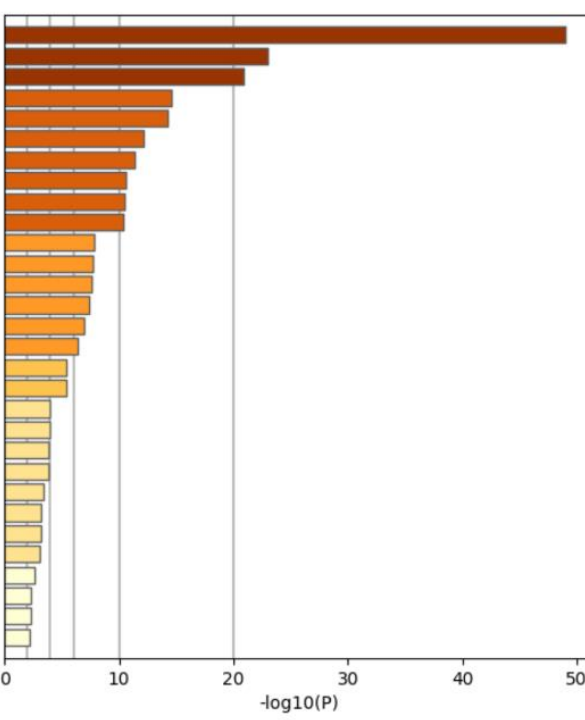

60:0051301: cell division

microtubule cytoskeleton organization involved in mitosis GO:0010564: regulation of cell cycle process

microtubules to kinetochore

O:1903046: meiotic cell cycle process

R-MMU-176408: Regulation of APC/C activators between G1/S and early anaphase -MMU-983189: Kinesins

GO:0000910: cytokinesis

(o chromosome, centromeric region

GO:0030261: chromosome condensation

protein serine/threonine kinase activity GO:0051653: spindle localization

GO:0007062: sister chromatid cohesion

R-MMU-606279: Deposition of new CENPA-containing nucleosomes at the centromere

GO:0006974: cellular response to DNA damage stimulus

GO:0070507: regulation of microtubule cytoskeleton organization

R-MMU-4615885: SUMOylation of DNA replication protein

GO:0006260: DNA replication

GO:0061351: neural precursor cell proliferation

GO:0072698: protein localization to microtubule cytoskeleton

00:007

GO:0031570: DNA integrity checkpoint

GO:0071901: negative regulation of protein serine/threonine kinase activity

GO:0071478: cellular response to radiation

GO:1903902: positive regulation of viral life cycle

GO:0050673: epithelial cell proliferation

GO:0008584: male gonad development

C


d
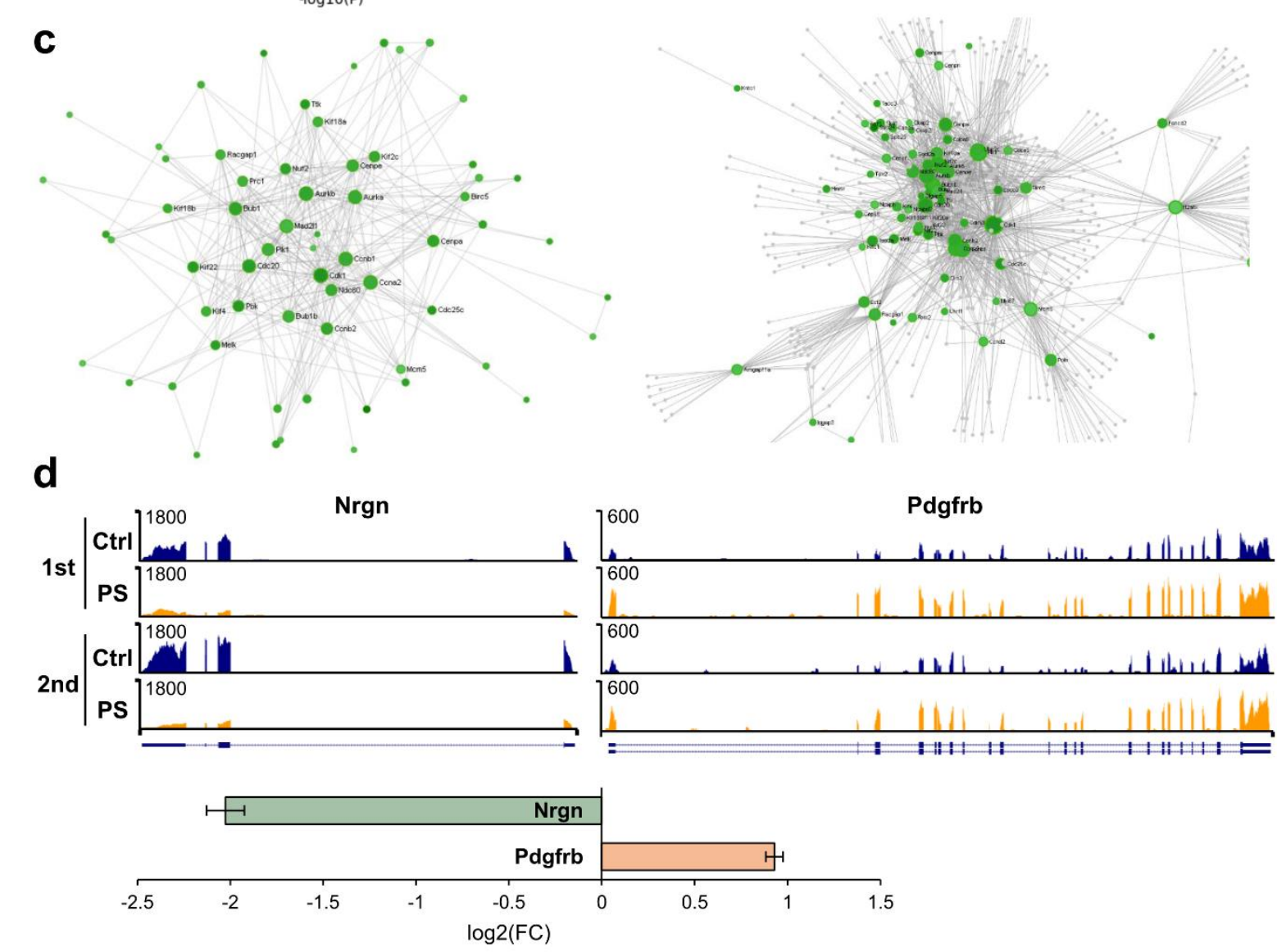
675 Figure 5. PSNPs altered the pattern of mRNA expression in mouse embryonic NSCs. (a)

676 Differential gene expression (DEG) analysis data show the genes commonly up- and down-regulated 677 by PSNPs ( $25 \mu \mathrm{g} / \mathrm{ml}$ carboxylated PSNPs of $50 \mathrm{~nm}$ diameter) in two biological replicates (analyzed by 678 Cuffdiff, FPKM FC > 1.5 and $p<0.05$ ). (b) Gene ontology analysis data show 125 genes (analyzed by 679 Metascape, $p<0.01)$ down-regulated by PSNPs. (c) Protein-protein interaction (PPI) network analysis 680 of 125 down-regulated genes by NetworkAnalyst with (left) or without (right) a "zero-order Network" 681 parameter. (d) mRNA-seq tracks show mRNA read abundance for Nrgn and Pdgfrb (upper). Two 682 biological replicates are shown. The average log2 (FC) values (Ctrl, PS) of the mRNAs are also shown 683 (lower).

684 
a
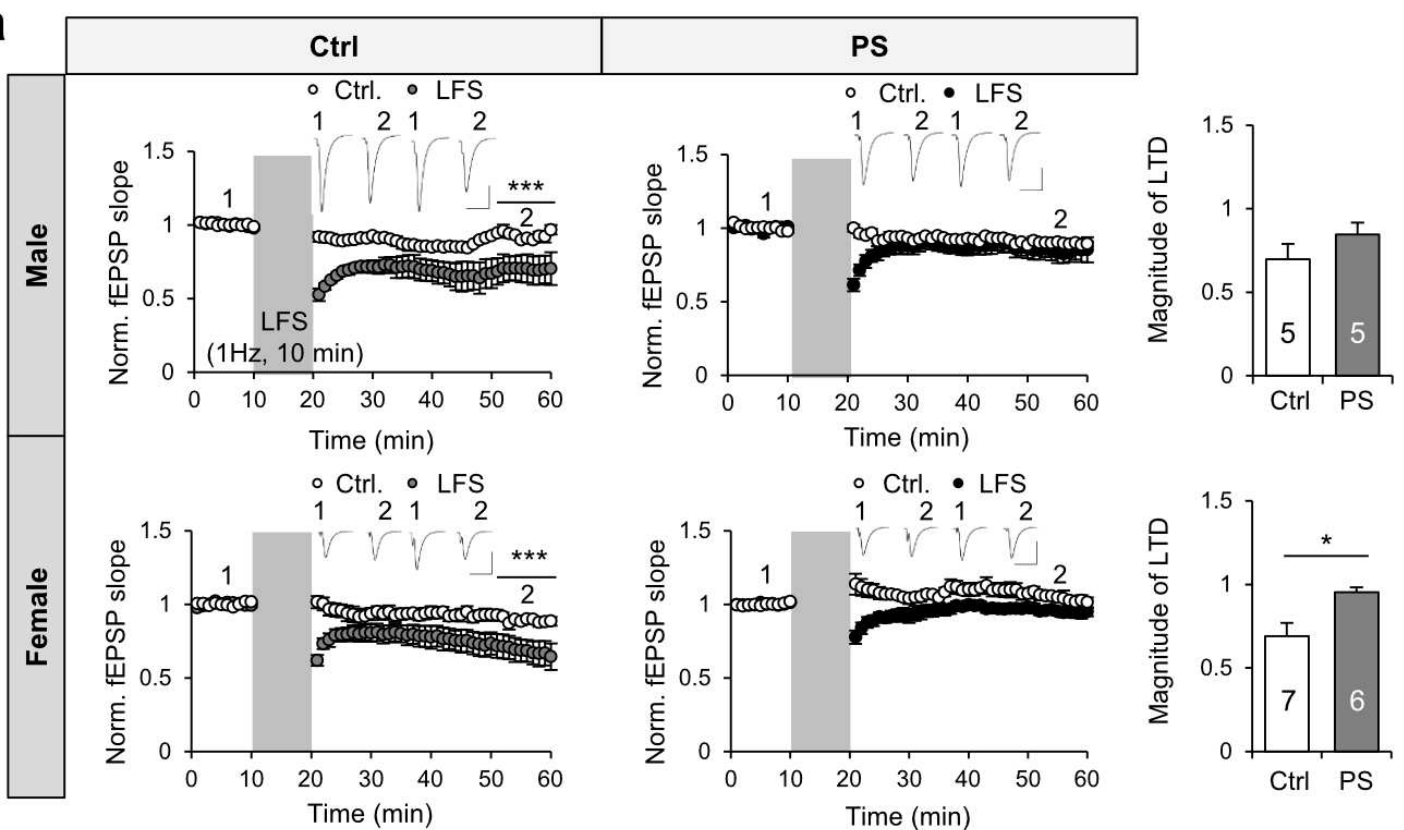

b
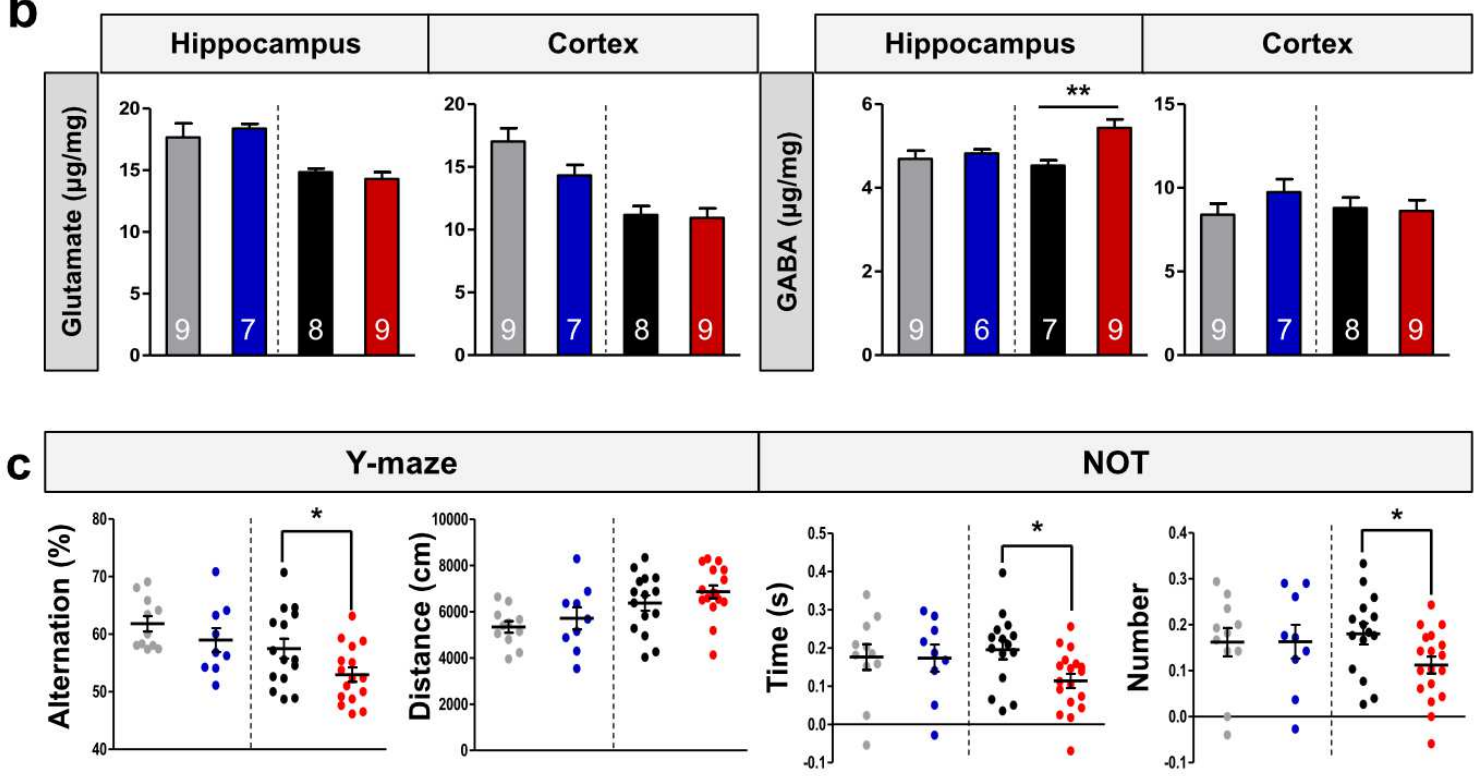

\begin{tabular}{|c|c|}
\hline OFT & Social interaction \\
\hline
\end{tabular}

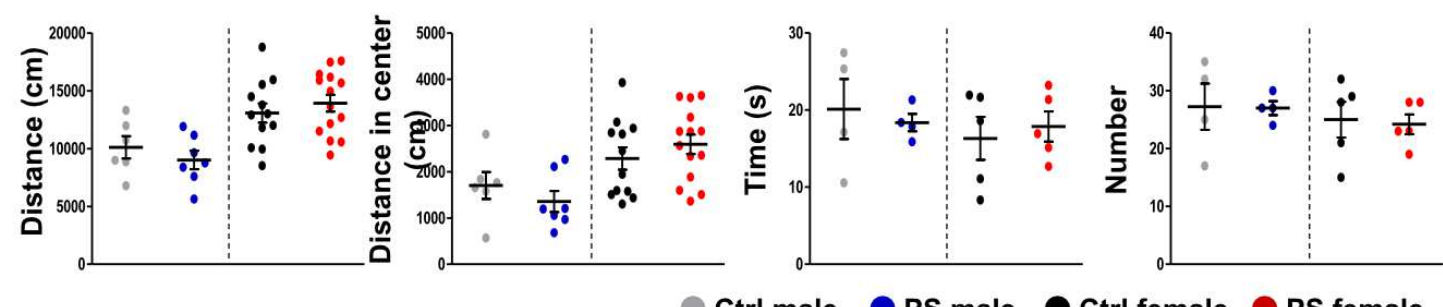


687 Figure 6. PSNPs caused neurophysiological abnormalities and cognitive impairment in female

688 progeny. (a) In comparison with baseline values, LFS decreased the rise slope of fEPSPs in control 689 mice (8-11 weeks old; males: $\mathrm{n}=5,70 \pm 9 \%, p<0.005$; females: $\mathrm{n}=7,69 \pm 8 \%, p<0.01$ ), but not in 690 PSNP-treated (500 $\mu$ g/day) mice (8-11 weeks old; males: $\mathrm{n}=5,85 \pm 7 \%, p>0.1$; females: $\mathrm{n}=7,95 \pm$ $6913 \%, p>0.1)$. The average magnitude of LTD induced by LFS for the rise slope was significantly different between control and PSNP-treated (500 $\mu \mathrm{g} /$ day) female mice $(p<0.05)$. Representative traces before 693 (1) and $35 \mathrm{~min}$ after (2) four episodes of TBS or LFS are shown. Scale bars: $20 \mathrm{~ms}, 0.2 \mathrm{mV}$. Values denote mean \pm SEM. ${ }^{*} p<0.05,{ }^{* *} p<0.01,{ }^{* * *} p<0.001$. (b) HPLC data show that a significant increase in the GABA level following PSNP exposure (500 $\mu \mathrm{g} /$ day) occurred only in the female hippocampus, with no change being observed in male mice. Values denote mean \pm SEM. ${ }^{*} p<0.05,{ }^{* *} p<0.005$. (c)

697 Y-maze test results show a female-specific decrease in alternation rate in the PSNP-treated (500 $\mu \mathrm{g} /$ day) group compared with controls. NOT data show a reduction of exploration time and frequency (number) specific to the female progeny following PSNP administration. Open field test (OFT) and social interaction test results show no change in either locomotion or social activity after PSNP administration. Values denote mean \pm SEM. ${ }^{*} p<0.05,{ }^{* *} p<0.005$ 


\section{Figures}

a

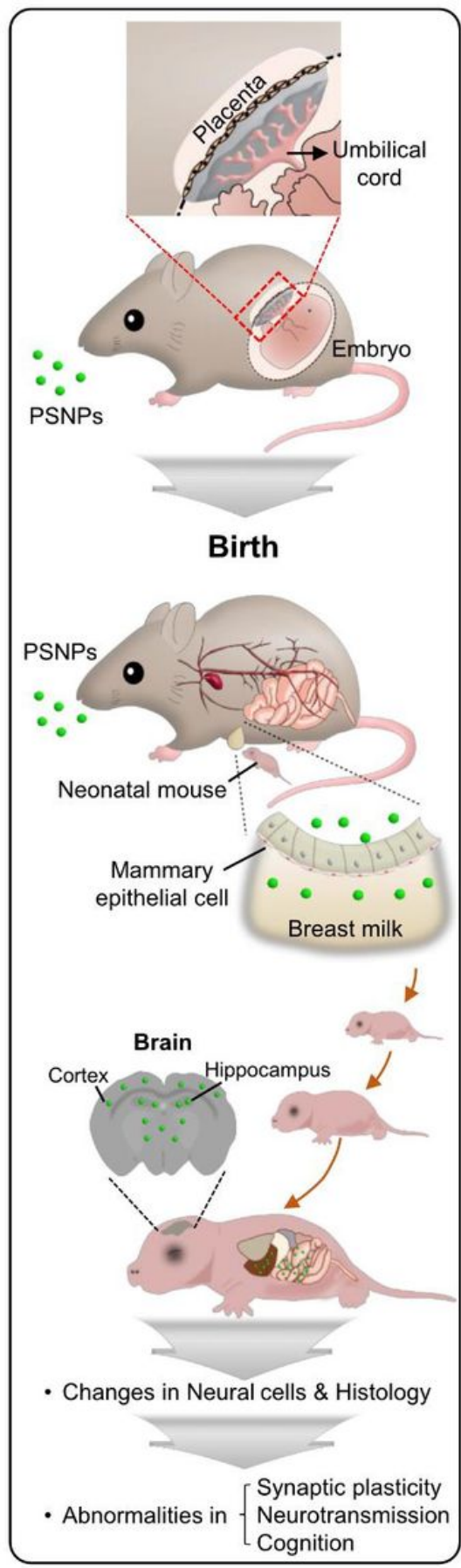

b
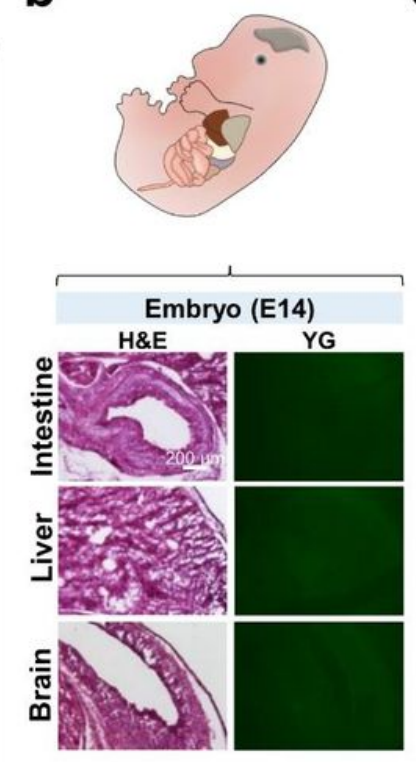

d

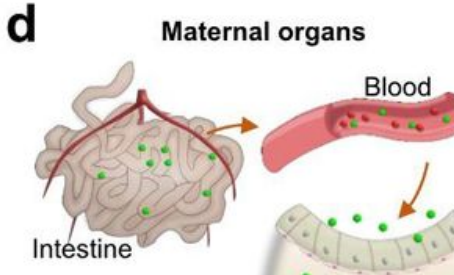

Mammary gland

e

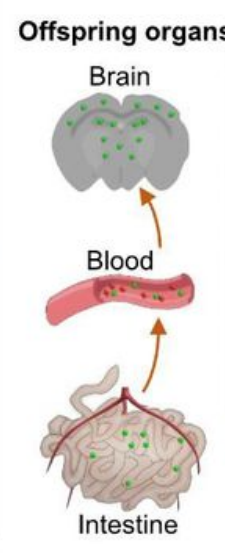

C
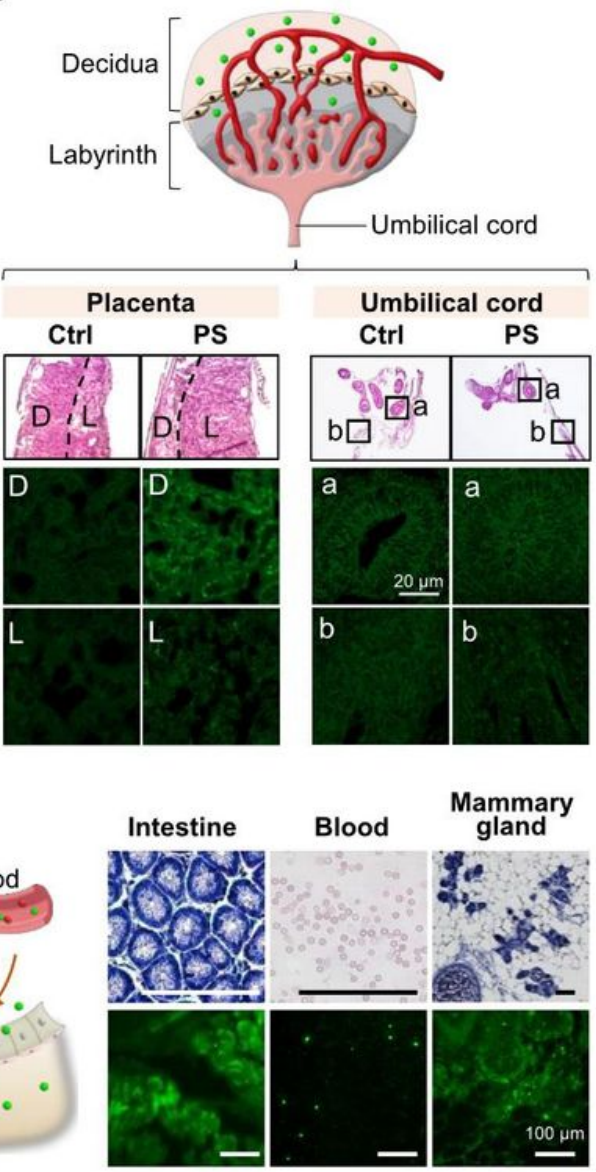

Brain

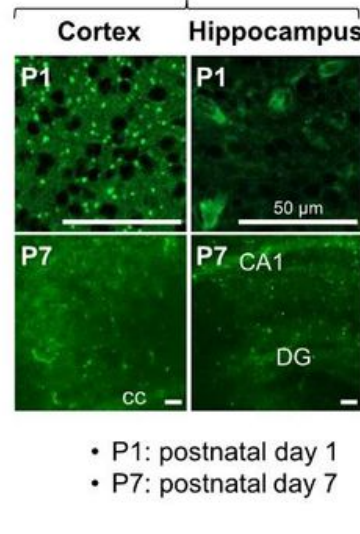

\section{Figure 1}

Maternally-administered PSNPs infiltrated various organs of the offspring and lactating mother. (a) Schematic drawing shows the overall experimental procedures. (b) The tissue analysis data show no YG fluorescence in embryonic tissues after maternal administration of PSNPs. (c) YG- conjugated PSNPs were detected in the maternal side of the placenta (decidua; D), but very few were detected in the fetal side of the placenta (labyrinth; L) after PSNP administration (PS). No fluorescence signal was observed in the umbilical cord of either control group (Ctrl) or PSNP treated group (PS). Histological structures were confirmed by H\&E staining. (d) Green 
fluorescence images show the accumulation of YG-conjugated PSNPs in maternal tissues including blood, intestines, and the liver. (e) YG-conjugated PSNPs were detected in the blood and organs of offspring at P1 and P7 including intestine, brain cortex, and hippocampus (cc: corpus callosum; DG: dentate gyrus).

a

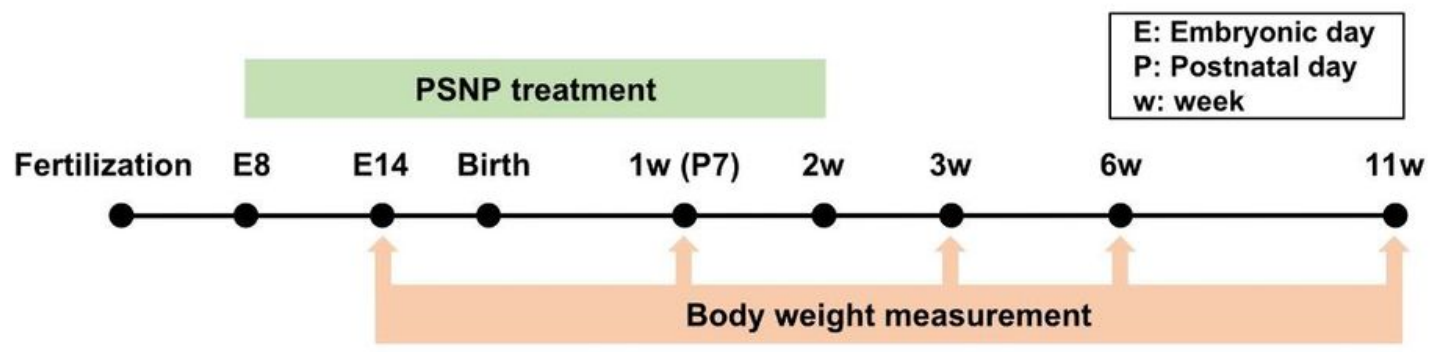

b
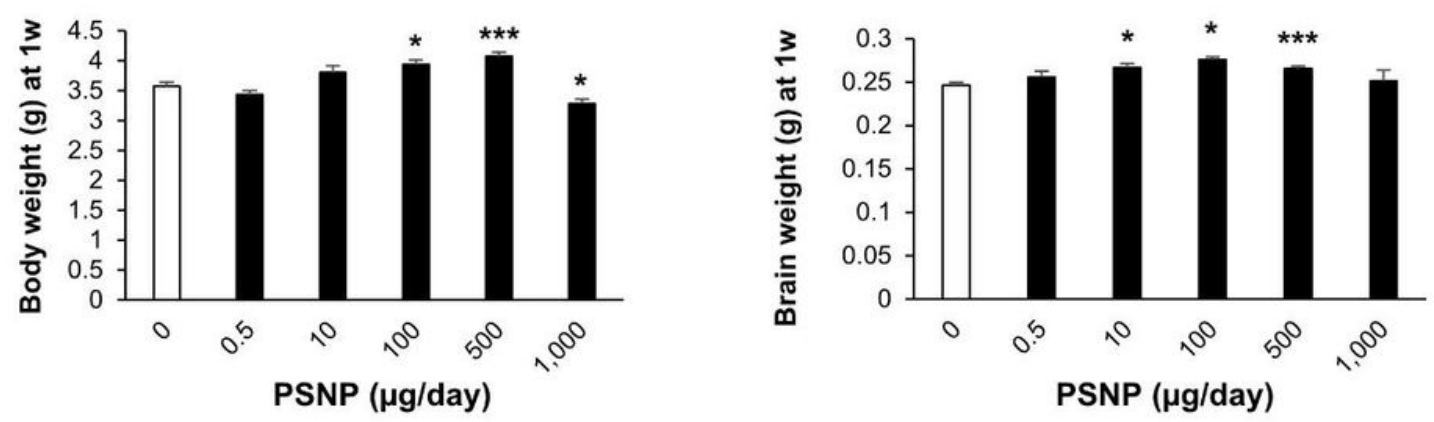

C
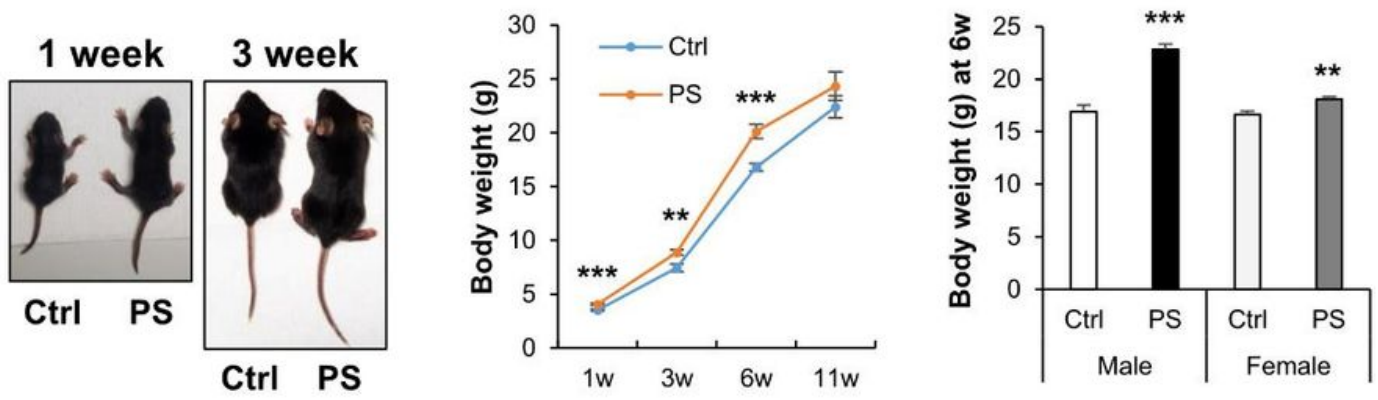

d
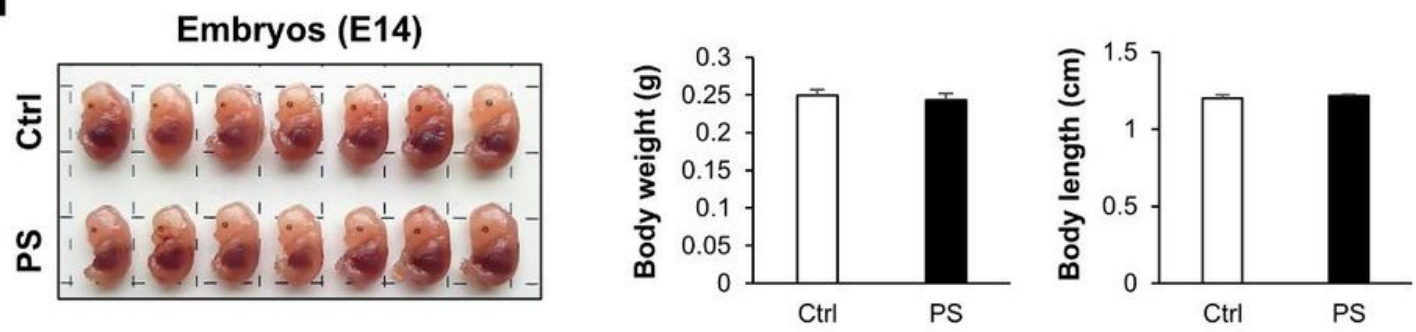

Figure 2

PSNPs increased the body and brain weights of the postnatal offspring. (a) Schematic drawing shows various time points at which body and brain weights were measured. (b) The PSNP-administered postnatal (1 w) group showed an increase in body weight and brain weight at doses of 100-500 $\mu \mathrm{g} /$ day. (c) The group that was administered $500 \mu \mathrm{g} /$ day of PSNPs (PS) showed the highest body weight increase at 6 weeks $(6 \mathrm{w})$, regardless of gender, with the difference not being any more significant at 11 weeks than in the controls (Ctrl). (d) No change in 
body weight or body length was observed in E14 embryos after PSNP treatment (PS). Values denote mean \pm SEM. ${ }^{*} p<0.05,{ }^{* *} p<6360.005,{ }^{* * *} p<0.0005$.

a

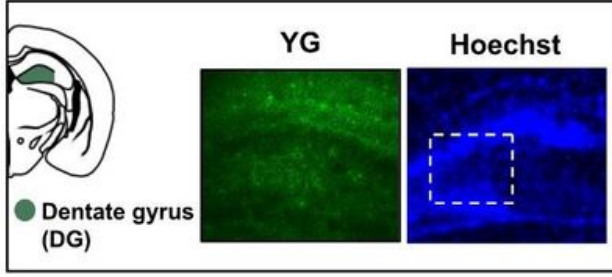

0
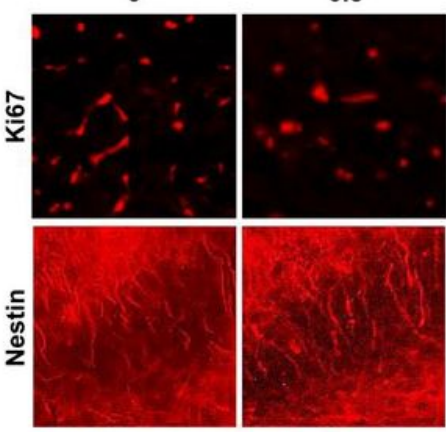

$\mathbf{C}$

b
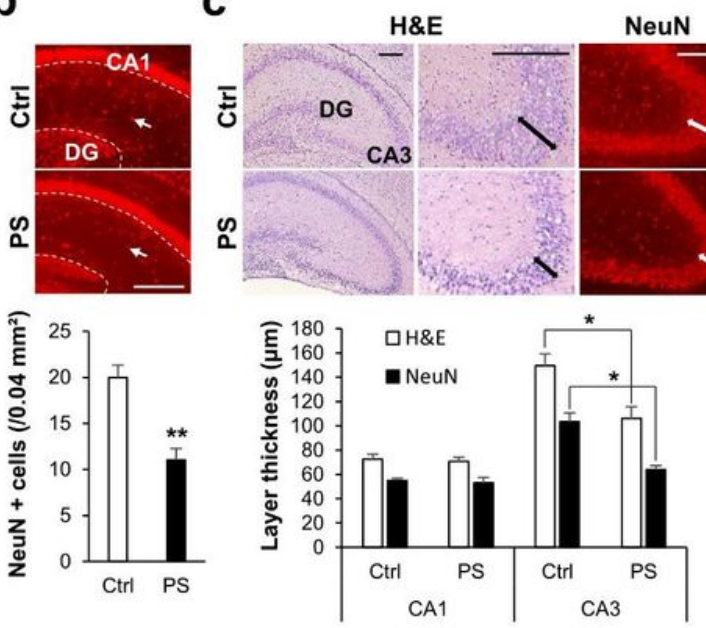

e
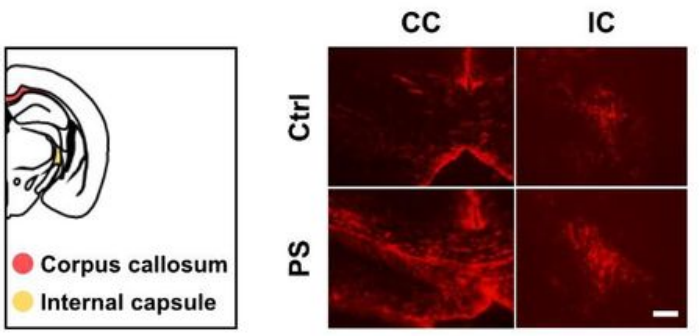

10

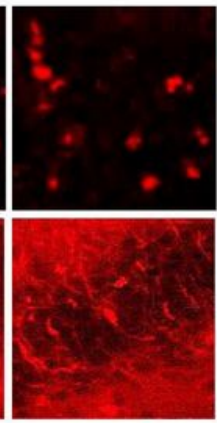

100

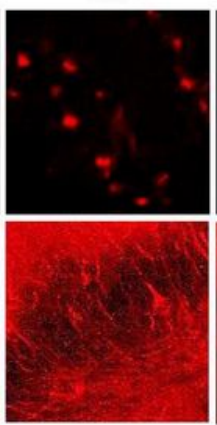

500

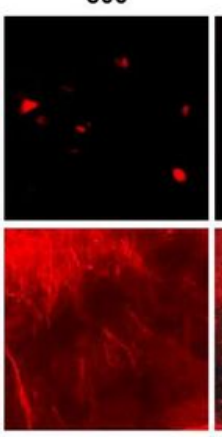

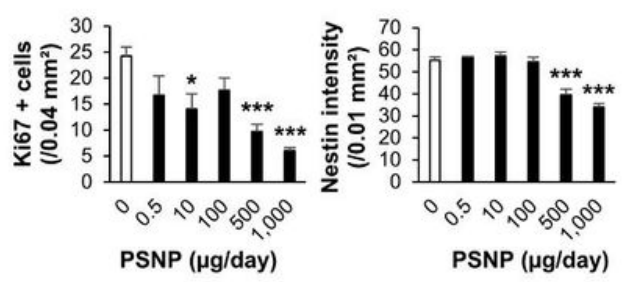

$1,000(\mu \mathrm{g} /$ day $)$



d
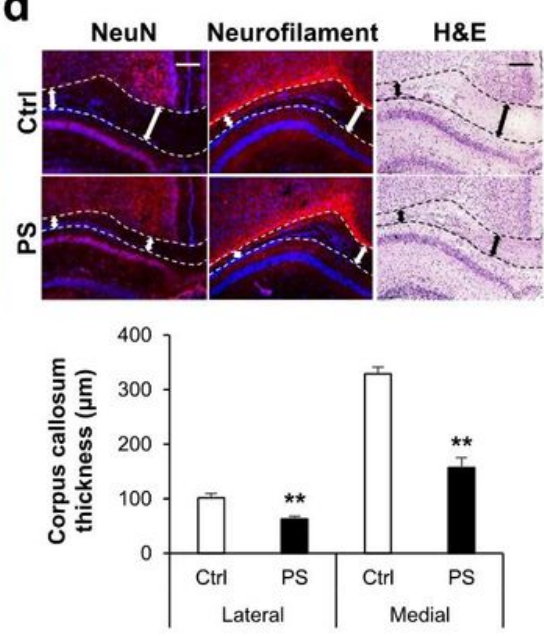

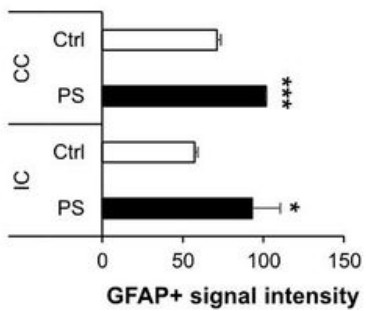

Figure 3

PSNPs altered neural cell composition in the brains of postnatal progeny. (a) The mouse brain atlas shows the location of the hippocampal dentate gyrus (DG). A green fluorescence image shows the accumulation of maternally-administered YG-conjugated carboxylated PSNPs (50-nm diameter; $500 \mu \mathrm{g} / \mathrm{day}$ ) in the hippocampus of the progeny at P7. Immunofluorescence staining results show that the number of Ki67+ and nestin+ progenitor cells was lower in the dentate gyrus of PSNP-exposed groups (carboxylated; 50-nm diameter) at $\geq 500 \mu \mathrm{g} / \mathrm{day}$. The number of Ki67+ cells and the nestin signal intensity are shown graphically. (b) Immunofluorescence staining 
data show decreased NeuN+ neurons in the stratum radiatum and stratum lacunosum-moleculare of the hippocampus (arrow) following PSNP administration (PS). (c) H\&E staining and NeuN immunolabeling data show reduced thickness of the hippocampal CA3 layer following $500 \mu \mathrm{g} /$ day PSNP exposure. (d) Immunolabeling and H\&E staining data show that the thickness of $\mathrm{NeuN}$ - and neurofilament+ corpus callosum was lower in mice exposed to $500 \mu \mathrm{g} /$ day PSNP treatment. (e) The mouse brain atlas shows the structure of the white matter including the corpus callosum (CC) and the internal capsule (IC). Immunofluorescence staining data show that the GFAP+ signal intensity in the white matter (CC and IC) was higher in mice exposed to $500 \mu \mathrm{g} /$ day of PSNPs. Values denote mean \pm SEM. ${ }^{*} p<0.05,{ }^{*} p<<0.005,{ }^{* *} \mathrm{p}<0.0005$. Scale bars: (b, c) $200 \mu \mathrm{m}$, (d, e) $100 \mu \mathrm{m}$.


C
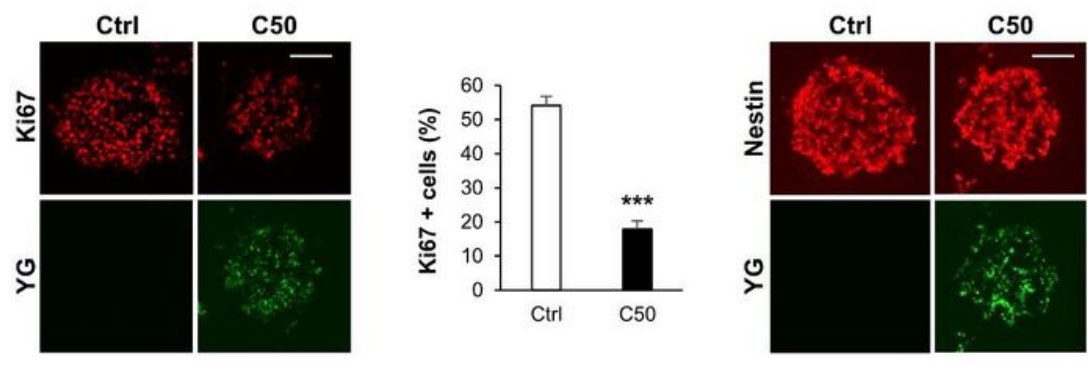

d

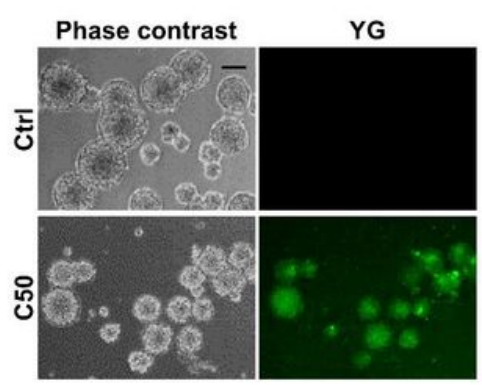

e
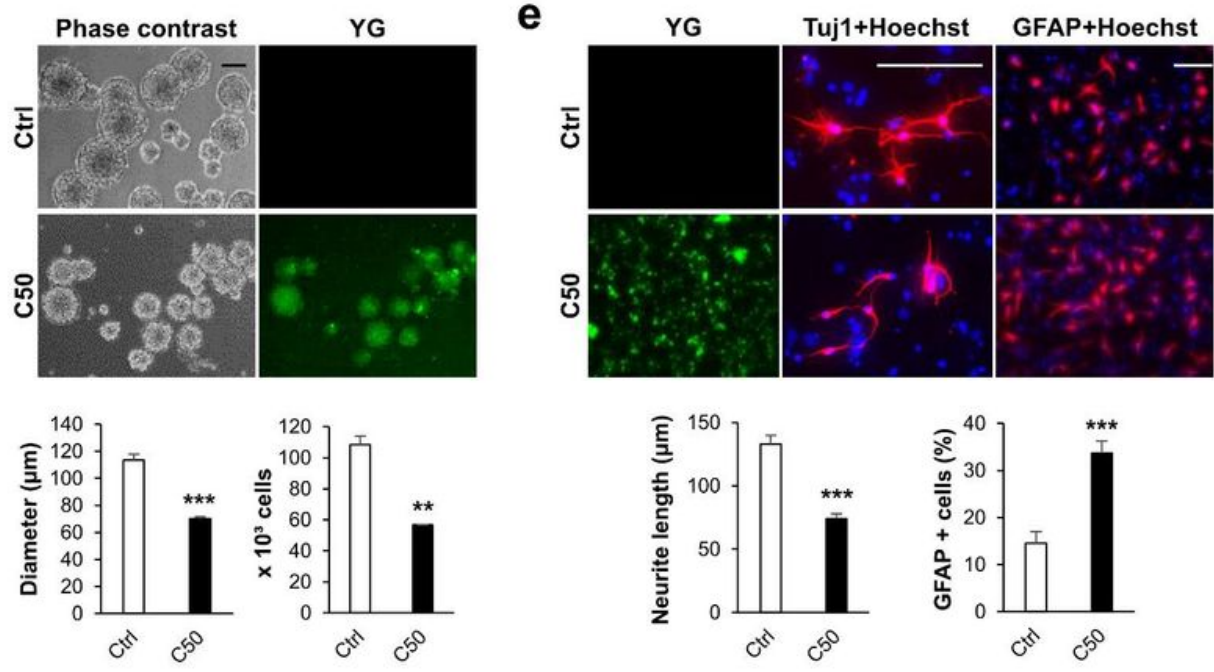

Figure 4 
PSNP exposure led to impaired proliferation and multi-lineage differentiation of NSCs in vitro. (a) An illustration summarizing the in vitro procedure used to examine the effects of PSNPs on NSC proliferation and multi-lineage differentiation. (b) The total cell number of NSCs was lower after exposure to $50 \mathrm{~nm}$ carboxylated PSNPs at $\geq 10$ $\mu \mathrm{g} / \mathrm{ml}$. (c) Immunofluorescence staining of single neurospheres shows a decrease in Ki67+ proliferative NSCs following exposure to PSNPs (C50;25 $\mu \mathrm{g} / \mathrm{ml}$ carboxylated PSNPs of $50 \mathrm{~nm}$ diameter). Other staining data show that nestin was well expressed in the neurospheres of the control (Ctrl) and PSNP-exposed groups (C50). YG fluorescence signals (green) were detected only in the PSNP-exposed group (C50). (d) Smaller neurosphere diameters and total cell numbers were observed in the PSNP-exposed group than in the controls (Ctrl). (e) NSC differentiation assay data show a decreased neurite length of Tuj1+ neurons and a significant increase in GFAP+ astrocytes in the $25 \mu \mathrm{g} / \mathrm{ml}$ PSNP-treated group compared with controls. C: carboxylated 670 PSNP; $50: 50 \mathrm{~nm}$. Values denote mean \pm SEM. ${ }^{\star \star} p<0.005$, ${ }^{\star \star \star} p<0.0005$. Scale bars: $100 \mu \mathrm{m}$. 
a

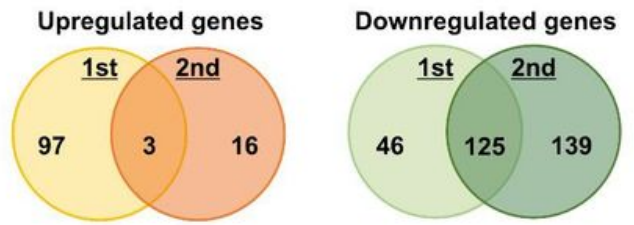

b
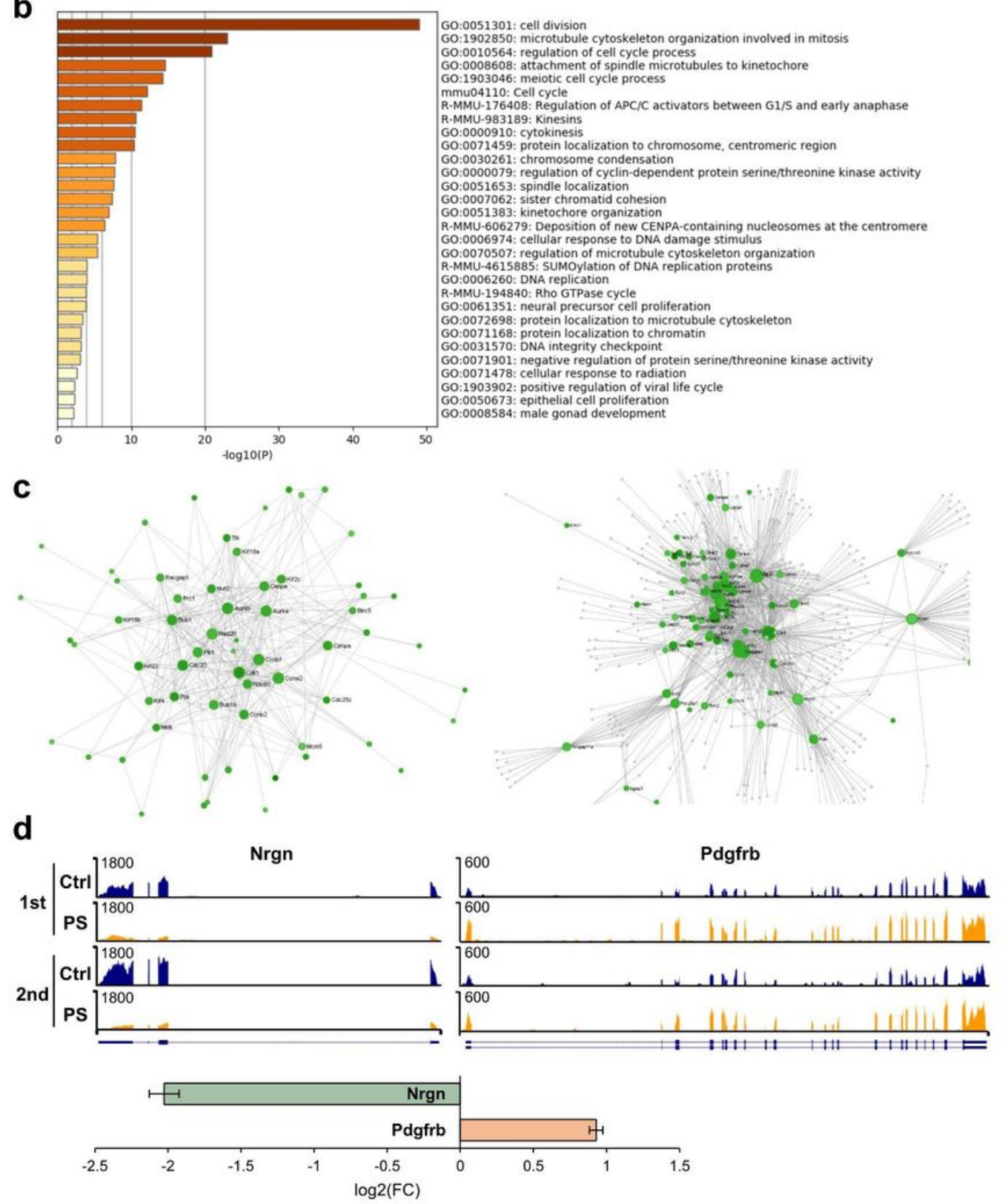

\section{Figure 5}

PSNPs altered the pattern of mRNA expression in mouse embryonic NSCs. (a) Differential gene expression (DEG) analysis data show the genes commonly up- and down-regulated by PSNPs $(25 \mu \mathrm{g} / \mathrm{ml}$ carboxylated PSNPs of 50 $\mathrm{nm}$ diameter) in two biological replicates (analyzed by Cuffdiff, FPKM FC > 1.5 and $\mathrm{p}<0.05$ ). (b) Gene ontology analysis data show 125 genes (analyzed by Metascape, $p<0.01$ ) down-regulated by PSNPs. (c) Protein-protein interaction (PPI) network analysis of 125 down-regulated genes by NetworkAnalyst with (left) or without (right) a "zero-order Network" parameter. (d) mRNA-seq tracks show mRNA read abundance for Nrgn and Pdgfrb (upper). Two biological replicates are shown. The average log2 (FC) values (Ctrl, PS) of the mRNAs are also shown (lower). 

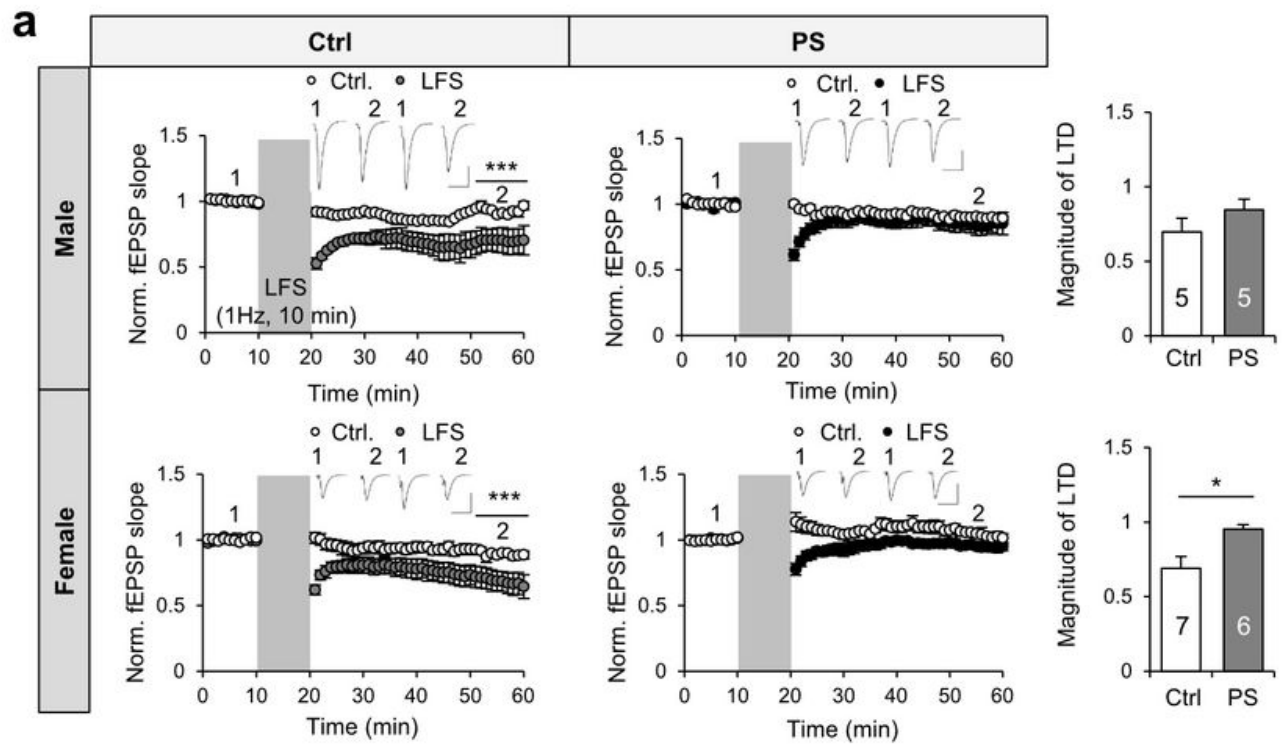

b
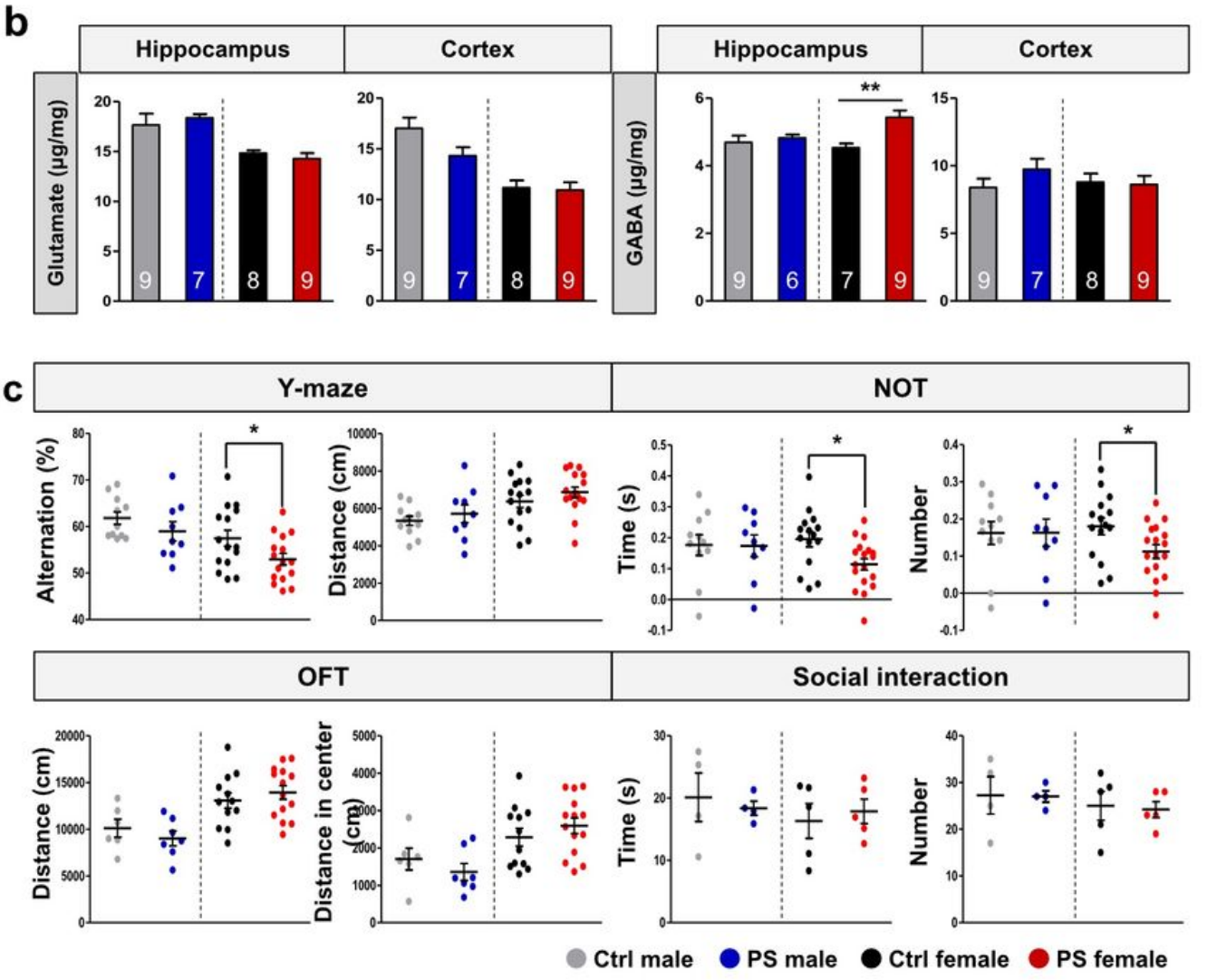

Figure 6

PSNPs caused neurophysiological abnormalities and cognitive impairment in female progeny. (a) In comparison with baseline values, LFS decreased the rise slope of fEPSPs in control (8-11 weeks old; males: $n=5,70 \pm 9 \%, p<$ 0.005; females: $n=7,69 \pm 8 \%, p<0.01)$, but not in PSNP-treated (500 $\mu \mathrm{g} /$ day) mice (8-11 weeks old; males: $n=5$, $85 \pm 7 \%, p>0.1$; females: $n=7,95 \pm 3 \%, p>0.1$ ). The average magnitude of LTD induced by LFS for the rise slope was significantly different between control and PSNP-treated $(500 \mu \mathrm{g} /$ day $)$ female mice $(p<0.05)$.

Representative traces before (1) and $35 \mathrm{~min}$ after (2) four episodes of TBS or LFS are shown. Scale bars: $20 \mathrm{ms,}$ $0.2 \mathrm{mV}$. Values denote mean \pm SEM. ${ }^{*} \mathrm{p}<0.05, * \star p<0.01, \star \star \star p p<0.001$. (b) HPLC data show that a significant increase in the GABA level following PSNP exposure ( $500 \mu \mathrm{g} /$ day) occurred only in the female hippocampus, with 
no change being observed in male mice. Values denote mean \pm SEM. ${ }^{*}<<0.05, * * p<0.005$. (c) Y-maze test results show a female-specific decrease in alternation rate in the PSNP-treated (500 $\mu \mathrm{g} /$ day) group compared with controls. NOT data show a reduction of exploration time and frequency (number) specific to the female progeny following PSNP administration. Open field test (OFT) and social interaction test results show no change in either locomotion or social activity after PSNP administration. Values denote mean \pm SEM. ${ }^{\star} p<0.05, * \star p<$ 0.005 .

\section{Supplementary Files}

This is a list of supplementary files associated with this preprint. Click to download.

- FNaturenanotechnology2020Jeongetal1218submissionfilesSupplementaryinformationNNANO20123137.pdf 